\title{
Fourth Moment Theorem and $q$-Brownian Chaos
}

\author{
Aurélien Deya], Salim Noreddinę2 and Ivan Nourdin 3
}

\begin{abstract}
In 2005, Nualart and Peccati [12] showed the so-called Fourth Moment Theorem asserting that, for a sequence of normalized multiple Wiener-Itô integrals to converge to the standard Gaussian law, it is necessary and sufficient that its fourth moment tends to 3. A few years later, Kemp et al. [8] extended this theorem to a sequence of normalized multiple Wigner integrals, in the context of the free Brownian motion. The $q$-Brownian motion, $q \in(-1,1]$, introduced by the physicists Frisch and Bourret [6] in 1970 and mathematically studied by Bożejko and Speicher [2] in 1991, interpolates between the classical Brownian motion $(q=1)$ and the free Brownian motion $(q=0)$, and is one of the nicest examples of non-commutative processes. The question we shall solve in this paper is the following: what does the Fourth Moment Theorem become when dealing with a $q$-Brownian motion?
\end{abstract}

Keywords: Central limit theorems; $q$-Brownian motion; non-commutative probability space; multiple integrals.

AMS subject classifications: 46L54; 60H05; 60F05

\section{INTRODUCTION AND MAIN RESULTS}

The $q$-Brownian motion was introduced in 1970 by the physicists Frisch and Bourret [6] as an intermediate model between two standard theoretical axiomatics (see also [7] for another physical interpretation). From a probabilistic point of view, it may be seen as a smooth and natural interpolation between two of the most fundamental processes in probability theory: on the one hand, the classical Brownian motion $\left(W_{t}\right)_{t \geq 0}$ defined on a classical probability space $(\Omega, \mathcal{F}, P)$; on the other hand, the free Brownian motion $\left(S_{t}\right)_{t \geq 0}$ at the core of Voiculescu's free probability theory and closely related to the study of large random matrices (see [14]).

The mathematical construction of the $q$-Brownian motion is due to Bożejko and Speicher [2, and it heavily relies on the theory of non-commutative probability spaces. Thus, before describing our results and for the sake of clarity, let us first introduce some of the central concepts of this theory (see [9] for a systematic presentation).

A $W^{*}$-probability space (or a non-commutative probability space) is a von Neumann algebra $\mathcal{A}$ (that is, an algebra of bounded operators on a real separable Hilbert space, closed under adjoint and convergence in the weak operator topology) equipped with a trace $\varphi$, that is, a unital linear functional (meaning preserving the identity) which is weakly continuous, positive (meaning $\varphi(X) \geq 0$ whenever $X$ is a non-negative element of $\mathcal{A}$; i.e. whenever $X=Y Y^{*}$ for some $Y \in \mathcal{A}$ ), faithful (meaning that if $\varphi\left(Y Y^{*}\right)=0$ then $Y=0$ ), and tracial (meaning that $\varphi(X Y)=\varphi(Y X)$ for all $X, Y \in \mathcal{A}$, even though in general $X Y \neq Y X)$.

\footnotetext{
${ }^{1}$ Institut Élie Cartan, Université de Lorraine, BP 70239, 54506 Vandoeuvre-lès-Nancy, France. Email: Aurelien.Deya@iecn.u-nancy.fr

${ }^{2}$ Laboratoire de Probabilités et Modèles Aléatoires, Université Paris 6, Boîte courrier 188, 4 Place Jussieu, 75252 Paris Cedex 5, France. Email: salim.noreddine@polytechnique.org

${ }^{3}$ Institut Élie Cartan, Université de Lorraine, BP 70239, 54506 Vandoeuvre-lès-Nancy, France. Email: inourdin@gmail.com. Supported in part by the two following (french) ANR grants: 'Exploration des Chemins Rugueux' [ANR-09-BLAN-0114] and 'Malliavin, Stein and Stochastic Equations with Irregular Coefficients' [ANR-10-BLAN-0121].
} 
In a $W^{*}$-probability space $(\mathcal{A}, \varphi)$, we refer to the self-adjoint elements of the algebra as random variables. Any random variable $X$ has a law: this is the unique compactly supported probability measure $\mu$ on $\mathbb{R}$ with the same moments as $X$; in other words, $\mu$ is such that

$$
\int_{\mathbb{R}} Q(x) d \mu(x)=\varphi(Q(X)),
$$

for any real polynomial $Q$. Thus, and as in the classical probability theory, the focus is more on the laws (which, in this context, is equivalent to the sequence of moments) of the random variables than on the underlying space $(\mathcal{A}, \varphi)$ itself. For instance, we say that a sequence $\left\{X_{k}\right\}_{k \geq 1}$ of random variables such that $X_{k} \in\left(\mathcal{A}_{k}, \varphi_{k}\right)$ converges to $X \in(\mathcal{A}, \varphi)$ if, for every positive integer $r$, one has $\varphi_{k}\left(X_{k}^{r}\right) \rightarrow \varphi\left(X^{r}\right)$ as $k \rightarrow \infty$. In the same way, we consider here that any family $\left\{X^{i}\right\}_{i \in I}$ of random variables on $(\mathcal{A}, \varphi)$ is 'characterized' by the set of all of its joint moments $\varphi\left(X^{i_{1}} \ldots X^{i_{r}}\right)\left(i_{1}, \ldots, i_{r} \in I, r \in \mathbb{N}\right)$, and we say that $\left\{X_{k}^{i}\right\}_{i \in I}$ converges to $\left\{X^{i}\right\}_{i \in I}$ (when $k \rightarrow \infty$ ) if the convergence of the joint moments holds true (see [9, Lecture 4] for further details on non-commutative random systems).

It turns out that a rather sophisticated combinatorial machinery is hidden behind most of these objects, see [9]. This leads in particular to the notion of crossing/non crossing pairing, which is a central tool in the theory.

Definition 1.1. 1. Let $r$ be an even integer. A pairing of $\{1, \ldots, r\}$ is any partition of $\{1, \ldots, r\}$ into $r / 2$ disjoint subsets, each of cardinality 2 . We denote by $\mathcal{P}_{2}(\{1, \ldots, r\})$ the set of all pairings of $\{1, \ldots, r\}$.

2. When $\pi \in \mathcal{P}_{2}(\{1, \ldots, r\})$, a crossing in $\pi$ is any set of the form $\left\{\left\{x_{1}, y_{1}\right\},\left\{x_{2}, y_{2}\right\}\right\}$ with $\left\{x_{i}, y_{i}\right\} \in \pi$ and $x_{1}<x_{2}<y_{1}<y_{2}$. The number of such crossings is denoted by $\operatorname{Cr}(\pi)$. The subset of all non-crossing pairings in $\mathcal{P}_{2}(\{1, \ldots, r\})$ (i.e., the subset of all $\pi \in \mathcal{P}_{2}(\{1, \ldots, r\})$ satisfying $\operatorname{Cr}(\pi)=0)$ is denoted by $N C_{2}(\{1, \ldots, r\})$.

By means of the objects given in Definition 1.1, it is simple to compute the joint moments related to the classical Brownian motion $W$ or to the free Brownian motion $S$, and this actually leads to the so-called Wick formula. Namely, for every $t_{1}, \ldots, t_{r} \geq 0$, one has

$$
\begin{aligned}
E\left[W_{t_{1}} \ldots W_{t_{r}}\right] & =\sum_{\pi \in \mathcal{P}_{2}(\{1, \ldots, r\})} \prod_{\{i, j\} \in \pi}\left(t_{i} \wedge t_{j}\right), \\
\varphi\left(S_{t_{1}} \ldots S_{t_{r}}\right) & =\sum_{\pi \in N C_{2}(\{1, \ldots, r\})} \prod_{\{i, j\} \in \pi}\left(t_{i} \wedge t_{j}\right) .
\end{aligned}
$$

It is possible to go smoothly from (2) to (3) by using the $q$-Brownian motion, which is one of the nicest examples of non-commutative processes.

Definition 1.2. Fix $q \in(-1,1)$. A q-Browian motion on some $W^{*}$-probability space $(\mathcal{A}, \varphi)$ is a collection $\left\{X_{t}\right\}_{t \geq 0}$ of random variables on $(\mathcal{A}, \varphi)$ satisfying that, for every integer $r \geq 1$ and every $t_{1}, \ldots, t_{r} \geq 0$,

$$
\varphi\left(X_{t_{1}} \ldots X_{t_{r}}\right)=\sum_{\pi \in \mathcal{P}_{2}(\{1, \ldots, r\})} q^{\mathrm{Cr}(\pi)} \prod_{\{i, j\} \in \pi}\left(t_{i} \wedge t_{j}\right) .
$$

The existence of such a process, far from being trivial, is ensured by the following result.

Theorem 1.3 (Bożejko, Speicher). For every $q \in(-1,1)$, there exists a $W^{*}$-probability space $\left(\mathcal{A}_{q}, \varphi_{q}\right)$ and a $q$-Brownian motion $\left\{X_{t}^{(q)}\right\}_{t \geq 0}$ built on it.

As is immediately seen, formula (4) allows to recover (2) by choosing $q=0$ (we adopt the usual convention $0^{0}=1$ ). On the other hand, although the classical Brownian motion $W$ cannot be identified with a process living on some $W^{*}$-probability space (the laws of its marginals being not 
compactly supported), it can legitimately be considered as the limit of $X^{(q)}$ when $q \rightarrow 1^{-}$. (This extension procedure can even be made rigorous by considering a larger class of non-commutative probability spaces.) As such, the family $\left\{X^{(q)}\right\}_{0 \leq q<1}$ of $q$-Brownian motions with a parameter $q$ between 0 and 1 happens to be a 'smooth' interpolation between $S$ and $W$.

Definition 1.4. Let $q \in(-1,1)$. For every $t \geq 0$, the distribution of $X_{t}^{(q)}$ is called the (centered) $q$-Gaussian law with variance $t$. We denote it by $\mathcal{G}_{q}(0, t)$. Otherwise stated, a given probability measure $\nu$ on $\mathbb{R}$ is distributed according to $\mathcal{G}_{q}(0, t)$ if it is compactly supported and if its moments are given by

$$
\int_{\mathbb{R}} x^{2 k+1} d \nu(x)=0 \quad \text { and } \quad \int_{\mathbb{R}} x^{2 k} d \nu(x)=t^{k} \sum_{\pi \in \mathcal{P}_{2}(\{1, \ldots, 2 k\})} q^{\mathrm{Cr}(\pi)} .
$$

The probability measure $\nu_{q} \sim \mathcal{G}_{q}(0,1)$ is absolutely continuous with respect to the Lebesgue measure; its density is supported by $\left[\frac{-2}{\sqrt{1-q}}, \frac{2}{\sqrt{1-q}}\right]$ and is given, within this interval, by

$$
\nu_{q}(d x)=\frac{1}{\pi} \sqrt{1-q} \sin \theta \prod_{n=1}^{\infty}\left(1-q^{n}\right)\left|1-q^{n} e^{2 i \theta}\right|^{2}, \quad \text { where } x=\frac{2 \cos \theta}{\sqrt{1-q}} \text { with } \theta \in[0, \pi] .
$$

By convention, we also set $\mathcal{G}_{1}(0, t)$ as being the probability measure whose density with respect to the Lebesgue measure is given by

$$
\frac{1}{\sqrt{2 \pi t}} e^{-\frac{x^{2}}{2 t}}, \quad x \in \mathbb{R}
$$

that is, $\mathcal{G}_{1}(0, t)=\mathcal{N}(0, t)$.

For every $q \in(-1,1)$, the process $X^{(q)}$ shares many similarities with the classical (resp. free) Brownian motion. For instance, it also appears as a limit process of some generalized random walks (see [3, Theorem 0]). For this reason, one sometimes considers this ' $q$-deformation' of $S$ and $W$ (see [2]). Also, and similarly to the free Brownian motion case, the $q$-Brownian motion appears as the limit of some particular sequences of $q$-Gaussian random variables (see [13]).

In the seminal paper [12, Nualart and Peccati highlighted a powerful convergence criterion for the normal approximation of sequences of multiple integrals with respect to the classical Brownian motion. From now on, we will refer to it as the Fourth Moment Theorem. A few years later, it was extended by Kemp et al. [8] for the free Brownian motion $S$ and its multiple Wigner integrals.

The question we shall solve in this paper is the following: what does the Fourth Moment Theorem become when dealing more generally with a $q$-Brownian motion? Before stating our main result and in order to put it into perspective, let us be more specific with the two aforementioned versions of the Fourth Moment Theorem that are already known (that is, in the classical and free Brownian motion cases). We let $I_{n}^{W}$ (resp. $I_{n}^{S}$ ) denote the $n$th multiple integrals with respect to $W$ (resp. $S$ ), as they are constructed in [1] (resp. [1]). The following two theorems are, respectively, the versions of the Fourth Moment Theorem in the classical case $(q=1)$ and in the free case $(q=0)$.

Theorem 1.5 (Nualart, Peccati). Fix $n \geq 2$ and let $\left\{f_{k}\right\}_{k \geq 1}$ be a sequence of symmetric functions in $L^{2}\left(\mathbb{R}_{+}^{n}\right)$ satisfying

$$
E\left[I_{n}^{W}\left(f_{k}\right)^{2}\right]=n !\left\|f_{k}\right\|_{L^{2}\left(\mathbb{R}_{+}^{n}\right)}^{2} \rightarrow 1 \quad \text { as } k \rightarrow \infty .
$$

Then, the following two assertions are equivalent as $k \rightarrow \infty$ :

(i) $E\left[I_{n}^{W}\left(f_{k}\right)^{4}\right] \rightarrow 3$.

(ii) The sequence $I_{n}^{W}\left(f_{k}\right)$ converges in law to $\mathcal{N}(0,1)=\mathcal{G}_{1}(0,1)$. 
Theorem 1.6 (Kemp, Nourdin, Peccati, Speicher). Fix $n \geq 2$ and let $\left\{f_{k}\right\}_{k \geq 1}$ be a sequence of mirror-symmetric functions in $L^{2}\left(\mathbb{R}_{+}^{n}\right)$ (that is, each $f_{k}$ is such that $f_{k}\left(t_{1}, \ldots, t_{n}\right)=f_{k}\left(t_{n}, \ldots, t_{1}\right)$ for almost all $\left.t_{1}, \ldots, t_{n} \geq 0\right)$ satisfying

$$
\varphi\left(I_{n}^{S}\left(f_{k}\right)^{2}\right)=\left\|f_{k}\right\|_{L^{2}\left(\mathbb{R}_{+}^{n}\right)}^{2} \rightarrow 1 \quad \text { as } k \rightarrow \infty .
$$

Then, the following two assertions are equivalent as $k \rightarrow \infty$ :

(i) $\varphi\left(I_{n}^{S}\left(f_{k}\right)^{4}\right) \rightarrow 2$.

(ii) The sequence $I_{n}^{S}\left(f_{k}\right)$ converges in law to $\mathcal{S}(0,1)=\mathcal{G}_{0}(0,1)$.

Now, fix a parameter $q \in[0,1]$, and consider a $q$-Brownian motion $X^{(q)}$ on some $W^{*}$ probability space $\left(\mathcal{A}_{q}, \varphi_{q}\right)$. (Note that in the three forthcoming statements, we extend the definition of $X^{(q)}$ to $q=1$ by naturally setting $X^{(1)}:=W$ and by replacing $\left(\mathcal{A}_{1}, \varphi_{1}\right)$ by $(\Omega, \mathcal{F}, P)$.) As in the classical and free cases, to each $n \geq 0$ we may associate with $X^{(q)}$ a natural notion of $n$th multiple integral $I_{n}^{X^{(q)}}$, see Donati-Martin [5] or Section 2.1 for the details. We are now in a position to state the main result of the present paper, which is a suitable interpolation between Theorem 1.5] and Theorem 1.6, but in a somehow unexpected way (see indeed the comment following its statement).

Theorem 1.7. Fix $n \geq 1$, recall that $q \in[0,1]$, and let $\left\{f_{k}\right\}_{k \geq 1}$ be a sequence of symmetric functions in $L^{2}\left(\mathbb{R}_{+}^{n}\right)$ satisfying

$$
\varphi_{q}\left(I_{n}^{X^{(q)}}\left(f_{k}\right)^{2}\right)=\left(\sum_{\sigma \in \mathfrak{S}_{n}} q^{\operatorname{inv}(\sigma)}\right)\left\|f_{k}\right\|_{L^{2}\left(\mathbb{R}^{n}\right)}^{2} \rightarrow 1 \quad \text { as } k \rightarrow \infty,
$$

where the notation $\operatorname{inv}(\sigma)$ refers to the number of inversions in $\sigma$, i.e.,

$$
\operatorname{inv}(\sigma):=\operatorname{Card}\{1 \leq i<j \leq n: \sigma(i)>\sigma(j)\} .
$$

Then the following two assertions are equivalent as $k \rightarrow \infty$ :

(i) $\varphi_{q}\left(I_{n}^{X^{(q)}}\left(f_{k}\right)^{4}\right) \rightarrow 2+q^{n^{2}}$.

(ii) The sequence $I_{n}^{X^{(q)}}\left(f_{k}\right)$ converges in law to $\mathcal{G}_{q^{n^{2}}}(0,1)$.

When, in Theorem 1.7, we consider a value of $q$ which is strictly between 0 and 1, we get that any suitably-normalized sequence $\left\{I_{n}^{X^{(q)}}\left(f_{k}\right)\right\}$ satisfying the fourth moment condition $(i)$ converges in law, see (ii), to a random variable which is expressed by means of the parameter $q^{n^{2}}$ and not $q$, as could have been legitimately expected by trying to guess the right statement with the help of Theorems 1.5 and 1.6. But this phenomenon was of course impossible to predict by taking a look at the case where $q \in\{0,1\}$ because, for these two values, we precisely have that $q=q^{n^{2}}$.

Two natural questions emerge from Theorem 1.7 $(a)$ what can be said when $q \in(-1,0)$ ? $(b)$ what happens if the functions $f_{k}$ are only mirror-symmetric (as in Theorem 1.6)? Regarding $(a)$, it is not difficult to build explicit counterexamples where the equivalence between $(i)$ and (ii) in Theorem 1.7 fails. For instance, with $q=-1 / 2, n=2$ and $f_{k}=f=\sqrt{2} \mathbf{1}_{[0,1]^{2}}$, we have $\varphi_{q}\left(I_{2}^{X^{(q)}}(f)^{2}\right)=1$ and $\varphi_{q}\left(I_{2}^{X^{(q)}}(f)^{4}\right)=2+q^{4}$, but $I_{2}^{X^{(q)}}(f)$ is not $\mathcal{G}_{q^{4}}(0,1)$-distributed (since $\left.\varphi_{q}\left(I_{2}^{X^{(q)}}(f)^{3}\right)=\sqrt{2}(1+q)^{2} \neq 0\right)$. See Remark 3.3 for the details. Actually, we do not know if this counterexample hides a general phenomenon or not. Does Theorem 1.7 continue to be true for all $q<0$ except (possibly) for some values of $q$, or is it always false when $q<0$ ? On the 
other hand, to answer question $(b)$ is unfortunately out of the scope of this paper. Indeed, to do so would imply to change almost all our computations (in order to take into account the lack of full symmetry). We postpone this further analysis to another paper.

Theorem 1.7will be obtained in Section 3 as a consequence of a more general multidimensional version (namely, Theorem 3.1). In fact, we will even prove that $(i)$ and $(i i)$ are both equivalent to a third assertion that only involves the sequence $\left\{f_{k}\right\}_{k \geq 1}$ and not the value of $q$ (provided it belongs to $[0,1])$. As a consequence, we shall deduce the following transfer principle.

Theorem 1.8 (Transfer principle). Fix $n \geq 1$ and let $\left\{f_{k}\right\}_{k \geq 1}$ be a sequence of symmetric functions in $L^{2}\left(\mathbb{R}_{+}^{n}\right)$ satisfying $\left\|f_{k}\right\|_{L^{2}\left(\mathbb{R}_{+}^{n}\right)}^{2} \rightarrow 1$ as $k \rightarrow \infty$. For every $q \in[0,1]$, set

$$
\sigma_{q}^{2}:=\sum_{\sigma \in \mathfrak{S}_{n}} q^{\operatorname{inv}(\sigma)}>0
$$

Then, the following two assertions are equivalent as $k \rightarrow \infty$ :

(i) The sequence $I_{n}^{X^{(q)}}\left(f_{k}\right)$ converges in law to $\mathcal{G}_{q^{n^{2}}}\left(0, \sigma_{q}^{2}\right)$ for one particular $q \in[0,1]$.

(ii) The sequence $I_{n}^{X^{(q)}}\left(f_{k}\right)$ converges in law to $\mathcal{G}_{q^{n^{2}}}\left(0, \sigma_{q}^{2}\right)$ for all $q \in[0,1]$.

As a nice application of all the previous material, we offer the following theorem. (We will prove it in Section 3.) For every $q \in[0,1]$, let us denote by $H_{0}^{(q)}, H_{1}^{(q)}, \ldots$ the sequence of $q$-Hermite polynomials, determined by the recurrence

$$
H_{0}^{(q)}(x)=1, \quad H_{1}^{(q)}(x)=x \quad \text { and } \quad x H_{n}^{(q)}(x)=H_{n+1}^{(q)}(x)+[n]_{q} H_{n-1}^{(q)},
$$

where $[n]_{q}=\frac{1-q^{n}}{1-q}\left(\right.$ with the convention that $\left.[n]_{1}=n\right)$. These polynomials are related to the $q$-Brownian motion $X^{(q)}$ through the formula

$$
H_{n}^{(q)}\left(I_{1}^{X^{(q)}}(e)\right)=I_{n}^{X^{(q)}}\left(e^{\otimes n}\right), \quad e \in L^{2}\left(\mathbb{R}_{+}\right), \quad\|e\|_{L^{2}\left(\mathbb{R}_{+}\right)}^{2}=1 .
$$

We then have:

Theorem 1.9 ( $q$-version of the Breuer-Major theorem). Fix $q \in[0,1]$ and let $n \geq 1$. Let $\left\{G_{l}\right\}_{l \in \mathbb{N}}$ be a q-Gaussian centered stationary family of random variables on some $W^{*}$-probability space $(\mathcal{A}, \varphi)$, meaning that there exists $\rho: \mathbb{Z} \rightarrow \mathbb{R}$ such that, for every integer $r \geq 1$ and every $l_{1}, \ldots, l_{r} \geq 1$, one has

$$
\varphi\left(G_{l_{1}} \ldots G_{l_{r}}\right)=\sum_{\pi \in \mathcal{P}_{2}(\{1, \ldots, r\})} q^{\mathrm{Cr}(\pi)} \prod_{\{a, b\} \in \pi} \rho\left(l_{a}-l_{b}\right) .
$$

Assume further that $\rho(0)=1$ (this just means that $G_{l} \sim \mathcal{G}_{q}(0,1)$ for every $l$ ) and $\sum_{l \in \mathbb{Z}}|\rho(l)|^{n}$ is finite. Then, as $k \rightarrow \infty$,

$$
\left\{\frac{1}{\sqrt{k}} \sum_{l=0}^{[k t]} H_{n}^{(q)}\left(G_{l}\right)\right\}_{t \geq 0} \stackrel{\text { f.d.d. }}{\rightarrow} \sqrt{\sum_{\sigma \in \mathfrak{S}_{n}} q^{\operatorname{inv}(\sigma)} \sum_{l \in \mathbb{Z}} \rho(l)^{n}}\left\{X_{t}^{\left(q^{n^{2}}\right)}\right\}_{t \geq 0},
$$

where 'f.d.d.' stands for the convergence in law of all finite-dimensional distributions and $X^{\left(q^{n^{2}}\right)}$ is a $q^{n^{2}}$-Brownian motion.

The rest of the paper is divided into two sections. In Section 2, we recall and prove some useful results relative to the so-called q-Gaussian chaos, which is nothing but a generalization of both the Wiener and Wigner chaoses. Notably, therein we extend the formula (4) to the case 
of multiple integrals with respect to the $q$-Brownian motion (Theorem 2.7). Once endowed with this preliminary material, we devote Section 3 to the proofs of Theorems 1.7, 1.8 and 1.9.

\section{2. $q$-BROWNIAN CHAOS AND PRODUCT FORMULAE}

Throughout this section, we fix a parameter $q \in(-1,1)$, as well as a $q$-Brownian motion $X^{(q)}$ on some $W^{*}$-probability space $\left(\mathcal{A}_{q}, \varphi_{q}\right)$. As a first step towards Theorem 1.7, our aim is to generalize the formula (44) to the case of multiple integrals with respect to $X^{(q)}$.

2.1. Multiple integrals. For every integer $n \geq 1$, the collection of all random variables of the type

$$
I_{n}^{X^{(q)}}(f)=\int_{\mathbb{R}_{+}^{n}} f\left(t_{1}, \ldots, t_{n}\right) d X_{t_{1}}^{(q)} \ldots d X_{t_{n}}^{(q)}, \quad f \in L^{2}\left(\mathbb{R}_{+}^{n}\right),
$$

is called the $n$th $q$-Gaussian chaos associated with $X^{(q)}$, and has been defined by Donati-Martin [5] along the same lines as the classical Wiener chaos (see, e.g., [11]), namely:

- first define $I_{n}^{X^{(q)}}(f)=\left(X_{b_{1}}^{(q)}-X_{a_{1}}^{(q)}\right) \ldots\left(X_{b_{n}}^{(q)}-X_{a_{n}}^{(q)}\right)$ when $f$ has the form

$$
f\left(t_{1}, \ldots, t_{n}\right)=\mathbf{1}_{\left(a_{1}, b_{1}\right)}\left(t_{1}\right) \times \ldots \times \mathbf{1}_{\left(a_{n}, b_{n}\right)}\left(t_{n}\right),
$$

where the intervals $\left(a_{i}, b_{i}\right), i=1, \ldots, n$, are pairwise disjoint;

- extend linearly the definition of $I_{n}^{X^{(q)}}(f)$ to the class $\mathcal{E}$ of simple functions vanishing on diagonals, that is, to functions $f$ that are finite linear combinations of indicators of the type (8);

- observe that, for all simple functions $f \in L^{2}\left(\mathbb{R}_{+}^{m}\right)$ and $g \in L^{2}\left(\mathbb{R}_{+}^{n}\right)$ vanishing on diagonals,

$$
\left\langle I_{m}^{X^{(q)}}(f), I_{n}^{X^{(q)}}(g)\right\rangle_{L^{2}\left(\mathcal{A}_{q}, \varphi_{q}\right)}=\varphi_{q}\left(I_{m}^{X^{(q)}}(f)^{*} I_{n}^{X^{(q)}}(g)\right)=\delta_{m, n}\langle f, g\rangle_{q},
$$

where the sesquilinear form $\langle., .\rangle_{q}$ is defined for all $f, g \in L^{2}\left(\mathbb{R}_{+}^{n}\right)$ by

$$
\langle f, g\rangle_{q}:=\sum_{\sigma \in \mathfrak{S}_{n}} q^{\operatorname{inv}(\sigma)} \int_{\mathbb{R}_{+}^{n}} f\left(t_{\sigma(1)}, \ldots, t_{\sigma(n)}\right) g\left(t_{1}, \ldots, t_{n}\right) d t_{1} \ldots d t_{n}
$$

and where $\delta_{m, n}$ stands for the Kronecker symbol;

- exploit the fact that the form $\langle., .\rangle_{q}$ is strictly positive on $L^{2}\left(\mathbb{R}_{+}^{n}\right)$ (see [2, Proposition 1]) in order to extend $I_{n}^{X^{(q)}}(f)$ to functions $f$ in the completion $\mathcal{F}_{q}$ of $\mathcal{E}$ with respect to $\langle., .\rangle_{q}$. Observe finally that, owing to the estimate $\|f\|_{q}^{2} \leq\left(\sum_{\sigma \in \mathfrak{S}_{n}} q^{\operatorname{inv}(\sigma)}\right)\|f\|_{L^{2}\left(\mathbb{R}_{+}^{n}\right)}^{2}$, one can rely on the inclusion $L^{2}\left(\mathbb{R}_{+}^{n}\right) \subset \mathcal{F}_{q}$ for every $q \in(-1,1)$ and every $n \geq 1$.

Of course, relation (9) continues to hold for every pair $f \in L^{2}\left(\mathbb{R}_{+}^{n}\right)$ and $g \in L^{2}\left(\mathbb{R}_{+}^{n}\right)$. Moreover, the above sketched construction implies that $I_{n}^{X^{(q)}}(f)$ is self-adjoint if and only if $f$ is mirror symmetric, i.e., $f^{*}=f$ where $f^{*}\left(t_{1}, \ldots, t_{n}\right):=f\left(t_{n}, \ldots, t_{1}\right)$.

Let us now report one of the main results of [5], namely the generalization of the product formula for multiple Wiener-Itô integrals to the $q$-Brownian motion case. In the sequel, we adopt the following notation.

Notation. With every $f \in L^{2}\left(\mathbb{R}_{+}^{n}\right)$ and every $p \in\{1, \ldots, n\}$, we associate the function $f_{q}^{(p)} \in L^{2}\left(\mathbb{R}_{+}^{n}\right)$ along the formula

$$
f_{q}^{(p)}\left(t_{1}, \ldots, t_{n-p}, s_{p}, \ldots, s_{1}\right):=\sum_{\sigma:\{1, \ldots, p\} \rightarrow\{1, \ldots, n\} \backslash} q^{\alpha(\sigma)} f\left(t_{1}, \ldots, s_{k}, \ldots, s_{1}, \ldots, t_{n-k}\right),
$$


where, in the right-hand-side, $\sigma$ is decreasing (this fact is written in symbols as $\sigma \searrow$ ), $s_{i}$ is at the place $\sigma(i)$, and

$$
\alpha(\sigma):=\sum_{i=1}^{p}(n+1-\sigma(i))-\frac{p(p+1)}{2} .
$$

Besides, we define another function $f_{q}^{[p]} \in L^{2}\left(\mathbb{R}_{+}^{n}\right)$ by

$$
f_{q}^{[p]}\left(s_{1}, \ldots, s_{p}, t_{1}, \ldots, t_{n-k}\right):=\sum_{\sigma:\{1, \ldots, p\} \rightarrow\{1, \ldots, n\}} q^{\beta(\sigma)} f\left(t_{1}, \ldots, s_{i}, \ldots t_{n-p}\right),
$$

where, in the right-hand-side, $s_{i}$ is at the place $\sigma(i)$ and

$$
\beta(\sigma):=\sum_{i=1}^{p} \sigma(i)-\frac{p(p+1)}{2}+\operatorname{inv}(\sigma) .
$$

(See Theorem 1.7 for the definition of $\operatorname{inv}(\sigma)$.)

We now introduce the central concept of contractions.

Definition 2.1. Fix $n, m \geq 1$ as well as $p \in\{1, \ldots, \min (m, n)\}$. Let $f \in L^{2}\left(\mathbb{R}_{+}^{n}\right)$ and $g \in$ $L^{2}\left(\mathbb{R}_{+}^{m}\right)$.

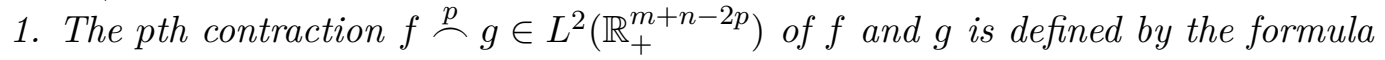

$$
\begin{aligned}
& f \stackrel{p}{\sim} g\left(t_{1}, \ldots, t_{m+n-2 p}\right) \\
& \quad=\int_{\mathbb{R}_{+}^{p}} f\left(t_{1}, \ldots, t_{n-p}, s_{p}, \ldots, s_{1}\right) g\left(s_{1}, \ldots, s_{p}, t_{n-p+1}, \ldots, t_{m+n-2 p}\right) d s_{1} \ldots d s_{p} .
\end{aligned}
$$

2. The pth $q$-contraction $f \stackrel{p}{q}_{q} g \in L^{2}\left(\mathbb{R}_{+}^{m+n-2 p}\right)$ of $f$ and $g$ is defined by the formula

$$
f \stackrel{p}{\stackrel{p}{q}_{q}} g=f_{q}^{(p)} \stackrel{p}{\stackrel{[p]}{q p}} .
$$

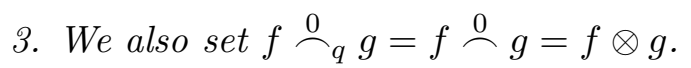

These contractions appear naturally in the product formula for multiple integrals with respect to the $q$-Brownian motion, that we state now.

Theorem 2.2 (Donati-Martin). Let $f \in L^{2}\left(\mathbb{R}_{+}^{n}\right)$ and $g \in L^{2}\left(\mathbb{R}_{+}^{m}\right)$ with $n, m \geq 1$. Then

$$
I_{n}^{X^{(q)}}(f) I_{m}^{X^{(q)}}(g)=\sum_{p=0}^{\min (n, m)} I_{n+m-2 p}^{X^{(q)}}\left(f \stackrel{p}{\sim}_{q} g\right) \text {. }
$$

2.2. Respecting pairings. As in [8], the notion of a respecting pairing will play a prominent role in our study.

Definition 2.3. Let $n_{1}, \ldots, n_{r}$ be positive integers and $n=n_{1}+\ldots+n_{r}$. The set $\{1, \ldots, n\}$ is then partitioned accordingly as $\{1, \ldots, n\}=B_{1} \cup B_{2} \cup \ldots \cup B_{r}$, where $B_{1}=\left\{1, \ldots, n_{1}\right\}$, $B_{2}=\left\{n_{1}+1, \ldots, n_{1}+n_{2}\right\}, \ldots, B_{r}=\left\{n_{1}+\ldots+n_{r_{1}}+1, \ldots, n\right\}$. We denote this partition by $n_{1} \otimes \ldots \otimes n_{r}$, and we will refer to the sets $B_{i}$ as the blocks of $n_{1} \otimes \ldots \otimes n_{r}$.

Then, we say that a pairing $\pi \in \mathcal{P}_{2}(\{1, \ldots, n\})$ respects $n_{1} \otimes \ldots \otimes n_{r}$ if every pair $\{l, m\} \in \pi$ is such that $l \in B_{i}$ and $m \in B_{j}$ with $i \neq j$. In the sequel, the subset of such respecting pairings in $\mathcal{P}_{2}(\{1, \ldots, n\})$ will be denoted as $C_{2}\left(n_{1} \otimes \ldots \otimes n_{r}\right)$. 
Finally, given $\pi \in C_{2}\left(n_{1} \otimes \ldots \otimes n_{r}\right)$ and functions $f^{1} \in L^{2}\left(\mathbb{R}_{+}^{n_{1}}\right), \ldots, f^{r} \in L^{2}\left(\mathbb{R}_{+}^{n_{r}}\right)$, we define the pairing integral

$$
\begin{aligned}
\int_{\pi} f_{1} & \otimes \ldots \otimes f_{r}:=\int_{\mathbb{R}_{+}^{n}} d t_{1} \ldots d t_{n} \\
& \times f_{1}\left(t_{1}, \ldots, t_{n_{1}}\right) f_{2}\left(t_{n_{1}+1}, \ldots, t_{n_{1}+n_{2}}\right) \ldots f_{r}\left(t_{n_{1}+\ldots+n_{r-1}+1}, \ldots, t_{n}\right) \prod_{\{i, j\} \in \pi} \delta\left(t_{i}-t_{j}\right),
\end{aligned}
$$

where $\delta$ stands for a Dirac mass at 0 .

For instance, consider the following pairing

$$
\pi:=\{(1,4),(2,8),(3,6),(5,9),(7,11),(10,12)\}
$$

as an element of $C_{2}(3 \otimes 4 \otimes 3 \otimes 2)$. Then it is readily checked that

$$
\int_{\pi} f_{1} \otimes f_{2} \otimes f_{3} \otimes f_{4}=\int_{\mathbb{R}_{+}^{6}} f_{1}\left(t_{1}, t_{2}, t_{3}\right) f_{2}\left(t_{1}, t_{4}, t_{3}, t_{5}\right) f_{3}\left(t_{2}, t_{4}, t_{6}\right) f_{4}\left(t_{5}, t_{6}\right) d t_{1} d t_{2} d t_{3} d t_{4} d t_{5} d t_{6} .
$$

Lemma 2.4. Let $f, g \in L^{2}\left(\mathbb{R}_{+}^{n}\right)$ and recall the definition (10) of $\langle f, g\rangle_{q}$. We have

$$
\langle f, g\rangle_{q}=\sum_{\sigma \in \mathfrak{S}_{n}} q^{\operatorname{inv}(\sigma)} \int_{P_{2}(\sigma)} f \otimes g^{*}
$$

where, for every $\sigma \in \mathfrak{S}_{n}$, the pairing $P_{2}(\sigma) \in C_{2}(n \otimes n)$ is explicitly given by

$$
P_{2}(\sigma):=\{(n+1-i, n+\sigma(i)), 1 \leq i \leq n\} .
$$

Proof. With each $\sigma \in \mathfrak{S}_{n}$, we may associate $\widetilde{\sigma} \in \mathfrak{S}_{n}$ given by $\widetilde{\sigma}(i)=n+1-\sigma(n+1-i)$. We then have, by the definition (12),

$$
\begin{aligned}
\int_{P_{2}(\widetilde{\sigma})} f \otimes g^{*} & =\int_{\mathbb{R}_{+}^{n}} f\left(s_{1}, \ldots, s_{n}\right) g^{*}\left(s_{n+1-\widetilde{\sigma}^{-1}(1)}, \ldots, s_{n+1-\widetilde{\sigma}^{-1}(n)}\right) d s_{1} \ldots d s_{n} \\
& =\int_{\mathbb{R}_{+}^{n}} f\left(s_{1}, \ldots, s_{n}\right) g\left(s_{n+1-\widetilde{\sigma}^{-1}(n)}, \ldots, s_{n+1-\widetilde{\sigma}^{-1}(1)}\right) d s_{1} \ldots d s_{n} \\
& =\int_{\mathbb{R}_{+}^{n}} f\left(t_{\sigma(1)}, \ldots, t_{\sigma(n)}\right) g\left(t_{\sigma\left(n+1-\widetilde{\sigma}^{-1}(n)\right)}, \ldots, t_{\sigma\left(n+1-\widetilde{\sigma}^{-1}(1)\right)}\right) d t_{1} \ldots d t_{n} .
\end{aligned}
$$

Now, we observe that $\sigma\left(n+1-\widetilde{\sigma}^{-1}(i)\right)=n+1-\widetilde{\sigma}\left(\widetilde{\sigma}^{-1}(i)\right)=n+1-i$, so that $\sigma(n+1-$ $\left.\tilde{\sigma}^{-1}(n+1-i)\right)=i$ for any $i$. We deduce that

$$
\int_{P_{2}(\widetilde{\sigma})} f \otimes g^{*}=\int_{\mathbb{R}_{+}^{n}} f\left(t_{\sigma(1)}, \ldots, t_{\sigma(n)}\right) g\left(t_{1}, \ldots, t_{n}\right) d t_{1} \ldots d t_{n} .
$$

Thus, since it is further readily checked that $\operatorname{inv}(\widetilde{\sigma})=\operatorname{inv}(\sigma)$ and that $\sigma \mapsto \widetilde{\sigma}$ is an involution, we get

$$
\begin{aligned}
\sum_{\sigma \in \mathfrak{S}_{n}} q^{\operatorname{inv}(\sigma)} \int_{P_{2}(\sigma)} f \otimes g^{*} & =\sum_{\sigma \in \mathfrak{S}_{n}} q^{\operatorname{inv}(\sigma)} \int_{P_{2}(\sigma)} f \otimes g^{*} \\
& =\sum_{\sigma \in \mathfrak{S}_{n}} q^{\operatorname{inv}(\sigma)} \int_{\mathbb{R}_{+}^{n}} f\left(t_{\sigma(1)}, \ldots, t_{\sigma(n)}\right) g\left(t_{1}, \ldots, t_{n}\right) d t_{1} \ldots d t_{n} \\
& =\langle f, g\rangle_{q} .
\end{aligned}
$$


2.3. Joint moments of multiple integrals. Let us eventually turn to the main concern of this section, that is, to the extension of (4) for multiple integrals $I_{n_{1}}^{X^{(q)}}\left(f^{1}\right), \ldots, I_{n_{r}}^{X^{(q)}}\left(f^{r}\right)$. To achieve this goal, we focus on the following construction procedure.

Fix some positive integers $n_{1}, \ldots, n_{r}$ with $r \geq 3$, as well as $p \in\left\{1, \ldots, \min \left(n_{1}, n_{2}\right)\right\}$. Then, given $\pi^{\prime} \in C_{2}\left(\left(n_{1}+n_{2}-2 p\right) \otimes n_{3} \otimes \ldots \otimes n_{r}\right), \sigma_{1}:\{1, \ldots, p\} \rightarrow\left\{1, \ldots, n_{1}\right\} \searrow$ and $\sigma_{2}:\{1, \ldots, p\} \rightarrow$ $\left\{1, \ldots, n_{2}\right\}$, we construct a pairing $\pi=F\left(\sigma_{1}, \sigma_{2}, \pi^{\prime}\right) \in C_{2}\left(n_{1} \otimes n_{2} \otimes \ldots \otimes n_{r}\right)$ as follows (see Figure 1 for an illustration):

1) In $\pi$, the first two blocks $\left\{1, \ldots, n_{1}\right\}$ and $\left\{n_{1}+1, \ldots, n_{1}+n_{2}\right\}$ are connected via exactly $p$ pairs given by

$$
\left\{\left(\sigma_{1}(i), n_{1}+\sigma_{2}(i)\right), 1 \leq i \leq p\right\} .
$$

2) The interactions between the $n_{1}+n_{2}-2 p$ remaining points in $\left\{1, \ldots, n_{1}+n_{2}\right\}$ and the set $\left\{n_{1}+n_{2}+1, \ldots, n_{1}+\ldots, n_{r}\right\}$, as well as the interactions within $\left\{n_{1}+n_{2}+1, \ldots, n_{1}+\ldots, n_{r}\right\}$, are governed along $\pi^{\prime}$.
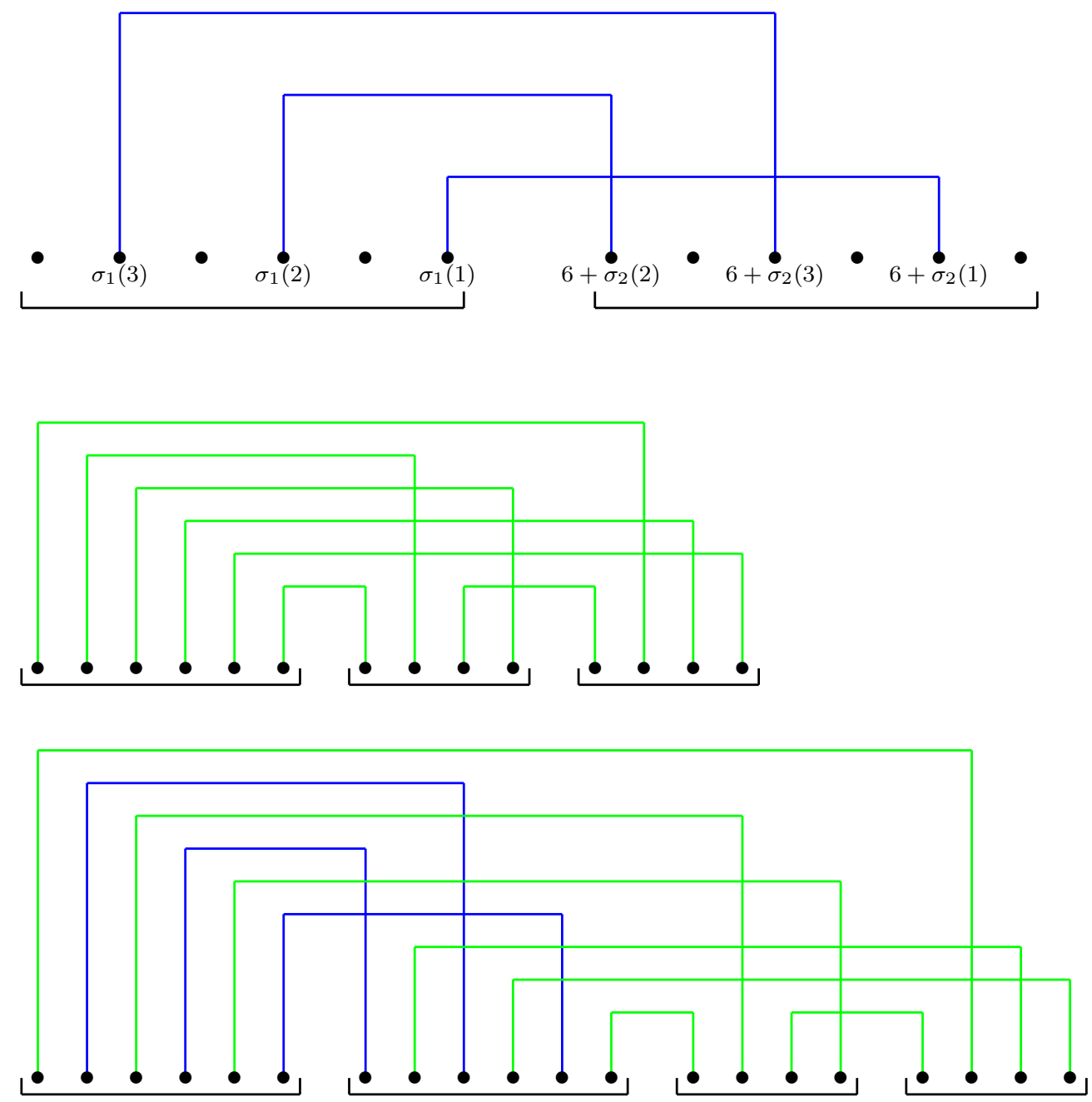

Figure 1. Construction of a pairing $\pi=F\left(\sigma_{1}, \sigma_{2}, \pi^{\prime}\right) \in C_{2}(6 \otimes 6 \otimes 4 \otimes 4)$ (third graph) from $\sigma_{1}:\{1,2,3\} \rightarrow\{1, \ldots, 6\} \searrow, \sigma_{2}:\{1,2,3\} \rightarrow\{1, \ldots, 6\}$ (first graph) and $\pi^{\prime} \in C_{2}(6 \otimes 4 \otimes 4)$ (second graph). 
This construction is clearly a one-to-one procedure. That is, given a pairing $\pi \in C_{2}\left(n_{1} \otimes n_{2} \otimes\right.$ $\left.\ldots \otimes n_{r}\right)$ such that the first two blocks $\left\{1, \ldots, n_{1}\right\}$ and $\left\{n_{1}+1, \ldots, n_{1}+n_{2}\right\}$ are linked by (exactly) $p$ pairs with $p \in\left\{1, \ldots, \min \left(n_{1}, n_{2}\right)\right\}$, there exists a unique $\sigma_{1}:\{1, \ldots, p\} \rightarrow\left\{1, \ldots, n_{1}\right\} \searrow$, a unique $\sigma_{2}:\{1, \ldots, p\} \rightarrow\left\{1, \ldots, n_{2}\right\}$ and a unique pairing $\pi^{\prime} \in C_{2}\left(\left(n_{1}+n_{2}-2 p\right) \otimes n_{3} \otimes \ldots \otimes n_{r}\right)$ such that $\pi=F\left(\sigma_{1}, \sigma_{2}, \pi^{\prime}\right)$. Besides, by the very definition of the $q$-contraction $f \stackrel{p}{q}_{q}$, the following result is easily checked:

Lemma 2.5. Fix $p \in\left\{1, \ldots, \min \left(n_{1}, n_{2}\right)\right\}$ and $\pi^{\prime} \in C_{2}\left(\left(n_{1}+n_{2}-2 p\right) \otimes n_{3} \otimes \ldots \otimes n_{r}\right)$. Then, for all functions $f^{1} \in L^{2}\left(\mathbb{R}_{+}^{n_{1}}\right), \ldots, f^{r} \in L^{2}\left(\mathbb{R}_{+}^{n_{r}}\right)$, one has

$$
\int_{\pi^{\prime}}\left(f_{1} \stackrel{p}{\rho_{q}} f_{2}\right) \otimes f_{3} \otimes \ldots \otimes f_{r}=\sum_{\substack{\sigma_{1}:\{1, \ldots, p\} \rightarrow\left\{1, \ldots, n_{1}\right\} \backslash \\ \sigma_{2}:\{1, \ldots, p\} \rightarrow\left\{1, \ldots, n_{2}\right\}}} q^{\alpha\left(\sigma_{1}\right)+\beta\left(\sigma_{2}\right)} \int_{F\left(\sigma_{1}, \sigma_{2}, \pi^{\prime}\right)} f_{1} \otimes f_{2} \otimes \ldots \otimes f_{r} .
$$

Our second ingredient for the generalization of (44) lies in the following computation of the crossings in $F\left(\sigma_{1}, \sigma_{2}, \pi^{\prime}\right)$ :

Lemma 2.6. Fix $p \in\left\{1, \ldots, \min \left(n_{1}, n_{2}\right)\right\}, \pi^{\prime} \in C_{2}\left(\left(n_{1}+n_{2}-2 p\right) \otimes n_{3} \otimes \ldots \otimes n_{r}\right), \sigma_{1}$ : $\{1, \ldots, p\} \rightarrow\left\{1, \ldots, n_{1}\right\} \searrow$ and $\sigma_{2}:\{1, \ldots, p\} \rightarrow\left\{1, \ldots, n_{2}\right\}$. Then

$$
\operatorname{Cr}\left(F\left(\sigma_{1}, \sigma_{2}, \pi^{\prime}\right)\right)=\alpha\left(\sigma_{1}\right)+\beta\left(\sigma_{2}\right)+\operatorname{Cr}\left(\pi^{\prime}\right) .
$$

Proof. Set $\pi:=F\left(\sigma_{1}, \sigma_{2}, \pi^{\prime}\right)$. The difference $D:=\operatorname{Cr}(\pi)-\operatorname{Cr}\left(\pi^{\prime}\right)$ is given by the number of crossings in $\pi$ that involve at least one of the pairs $\left\{\left(\sigma_{1}(i), n_{1}+\sigma_{2}(i)\right), 1 \leq i \leq p\right\}$. In order to compute this quantity, consider the following iterative procedure:

- Step 1: Compute the number of crossings that involve the pair $\left(\sigma_{1}(1), n_{1}+\sigma_{2}(1)\right)$. Since $\sigma_{1}$ is decreasing and $\pi \in C_{2}\left(n_{1} \otimes \ldots \otimes n_{r}\right)$, this is just the number of points between $\sigma_{1}(1)$ and $n_{1}+\sigma_{2}(1)$, i.e., $\left(n_{1}+\sigma_{2}(1)\right)-\sigma_{1}(1)-1$.

- Step 2: Compute the number of crossings that involve the pair $\left(\sigma_{1}(2), n_{1}+\sigma_{2}(2)\right)$, leaving aside the possible crossings between $\left(\sigma_{1}(2), n_{1}+\sigma_{2}(2)\right)$ and $\left(\sigma_{1}(1), n_{1}+\sigma_{2}(1)\right)$ (they have already been taken into account in Step 1). Since $\sigma_{1}(1)>\sigma_{1}(2)$, this leads to $\left(n_{1}+\sigma_{2}(2)\right)-\sigma_{1}(2)-2-$ $1_{\left\{\sigma_{2}(1)<\sigma_{2}(2)\right\}}$ crossings.

- Step l: Compute the number of crossings that involve the pair $\left(\sigma_{1}(l), n_{1}+\sigma_{2}(l)\right)$, leaving aside the possible crossings between $\left(\sigma_{1}(l), n_{1}+\sigma_{2}(l)\right)$ and $\left(\sigma_{1}(1), n_{1}+\sigma_{2}(1)\right), \ldots,\left(\sigma_{1}(l-1), n_{1}+\right.$ $\sigma_{2}(l-1)$ ) (they have already been taken into account in the previous steps). This yields $\left(n_{1}+\right.$ $\left.\sigma_{2}(l)\right)-\sigma_{1}(l)-l-\sum_{j=1}^{l-1} 1_{\left\{\sigma_{2}(j)<\sigma_{2}(l)\right\}}$ crossings. 
By repeating this procedure up to Step $p$, one can compute $D$ as follows:

$$
\begin{aligned}
D & =\sum_{l=1}^{p}\left[\left(n_{1}+\sigma_{2}(l)\right)-\sigma_{1}(l)-l-\sum_{j=1}^{l-1} 1_{\left\{\sigma_{2}(j)<\sigma_{2}(l)\right\}}\right] \\
& =\sum_{l=1}^{p}\left[\left(n_{1}+\sigma_{2}(l)\right)-\sigma_{1}(l)\right]-\left\{\frac{p(p+1)}{2}+\sum_{l=1}^{p} \sum_{j=1}^{l-1} 1_{\left\{\sigma_{2}(j)<\sigma_{2}(l)\right\}}\right\} \\
& =\sum_{l=1}^{p}\left[\left(n_{1}+\sigma_{2}(l)\right)-\sigma_{1}(l)\right]-\left\{p^{2}-\operatorname{inv}\left(\sigma_{2}\right)\right\} \\
& =\left[p\left(n_{1}+1\right)-\sum_{l=1}^{p} \sigma_{1}(l)-\frac{p(p+1)}{2}\right]+\left[\sum_{l=1}^{p} \sigma_{2}(l)-\frac{p(p+1)}{2}+\operatorname{inv}\left(\sigma_{2}\right)\right] \\
& =\alpha\left(\sigma_{1}\right)+\beta\left(\sigma_{2}\right) .
\end{aligned}
$$

We are now in a position to state the main result of this section, which is a suitable generalization of (44). (We recover (44) by choosing $n_{1}=\ldots=n_{r}=1$ and $f_{i}=\mathbf{1}_{\left[0, t_{i}\right]}, i=1, \ldots, r$.)

Theorem 2.7. Let $n_{1}, \ldots, n_{r}$ be positive integers. For all functions $f_{1} \in L^{2}\left(\mathbb{R}_{+}^{n_{1}}\right), \ldots, f_{r} \in$ $L^{2}\left(\mathbb{R}_{+}^{n_{r}}\right)$, it holds that

$$
\varphi_{q}\left(I_{n_{1}}^{X^{(q)}}\left(f_{1}\right) \ldots I_{n_{r}}^{X^{(q)}}\left(f_{r}\right)\right)=\sum_{\pi \in C_{2}\left(n_{1} \otimes \ldots \otimes n_{r}\right)} q^{\mathrm{Cr}(\pi)} \int_{\pi} f_{1} \otimes \ldots \otimes f_{r} .
$$

Proof. The proof is by induction on $r \geq 2$. For $r=2$, observe first that $C_{2}\left(n_{1} \otimes n_{2}\right) \neq \emptyset \Leftrightarrow n_{1}=$ $n_{2}$. Then, with the notation of Section 2.2, every $\pi \in C_{2}(n \otimes n)$ can be written as $\pi=P_{2}(\sigma)$ for a unique $\sigma \in \mathfrak{S}_{n}$, and one has $\operatorname{Cr}(\pi)=\operatorname{inv}(\sigma)$ since, for every $i, j \in\{1, \ldots, n\}$,

$$
n+1-j<n+1-i<n+\sigma(j)<n+\sigma(i) \quad \Longleftrightarrow \quad i<j \text { and } \sigma(j)<\sigma(i) .
$$

Therefore, according to (13) and (9),

$$
\sum_{\pi \in C_{2}(n \otimes n)} q^{\operatorname{Cr}(\pi)} \int_{\pi} f_{1} \otimes f_{2}=\sum_{\sigma \in \mathfrak{S}_{n}} q^{\operatorname{inv}(\sigma)} \int_{P_{2}(\sigma)} f_{1} \otimes f_{2}=\left\langle f_{1}, f_{2}^{*}\right\rangle_{q}=\varphi_{q}\left(I_{n}^{X^{(q)}}\left(f_{1}\right) I_{n}^{X^{(q)}}\left(f_{2}\right)\right),
$$

which corresponds to (16) in this case.

Assume now that (16) holds true for every $\left(n_{1}, \ldots, n_{s}\right)$ with $s \leq r-1(r \geq 3)$, and fix $n_{1}, \ldots, n_{r} \geq$ 1. According to the product formula (11), one can write

$$
\varphi_{q}\left(I_{n_{1}}^{X^{(q)}}\left(f_{1}\right) \ldots I_{n_{r}}^{X^{(q)}}\left(f_{r}\right)\right)=\sum_{p=0}^{\min \left(n_{1}, n_{2}\right)} \varphi_{q}\left(I_{n_{1}+n_{2}-2 p}^{X^{(q)}}\left(f_{1} \stackrel{p}{\stackrel{p}{q}_{q}} f_{2}\right) I_{n_{3}}^{X^{(q)}}\left(f_{3}\right) \ldots I_{n_{r}}^{X^{(q)}}\left(f_{r}\right)\right),
$$

and hence, by our induction assumption,

$\varphi_{q}\left(I_{n_{1}}^{X^{(q)}}\left(f_{1}\right) \ldots I_{n_{r}}^{X^{(q)}}\left(f_{r}\right)\right)=\sum_{p=0}^{\min \left(n_{1}, n_{2}\right)} \sum_{\pi \in C_{2}\left(\left(n_{1}+n_{2}-2 p\right) \otimes n_{3} \otimes \ldots \otimes n_{r}\right)} q^{\operatorname{Cr}(\pi)} \int_{\pi}\left(f_{1} \stackrel{p}{q}_{q} f_{2}\right) \otimes f_{3} \otimes \ldots \otimes f_{r}$.

Now, given $\pi \in C_{2}\left(n_{1} \otimes \ldots \otimes n_{r}\right)$, denote by $p_{\pi} \in\left\{0, \ldots, \min \left(n_{1}, n_{2}\right)\right\}$ the number of pairs in $\pi$ that link the first two blocks $\left\{1, \ldots, n_{1}\right\}$ and $\left\{n_{1}+1, \ldots, n_{1}+n_{2}\right\}$. By using successively 
Formulae (14) and (15), we deduce

$$
\begin{aligned}
& \varphi_{q}\left(I_{n_{1}}^{X^{(q)}}\left(f_{1}\right) \ldots I_{n_{r}}^{X^{(q)}}\left(f_{r}\right)\right) \\
& =\sum_{\pi \in C_{2}\left(\left(n_{1}+n_{2}\right) \otimes n_{3} \otimes \ldots \otimes n_{r}\right)} q^{\operatorname{Cr}(\pi)} \int_{\pi}\left(f_{1} \otimes f_{2}\right) \otimes f_{3} \otimes \ldots \otimes f_{r} \\
& +\sum_{p=1}^{\min \left(n_{1}, n_{2}\right)} \sum_{\substack{\pi \in C_{2}\left(\left(n_{1}+n_{2}-2 p\right) \otimes n_{3} \otimes \ldots \otimes n_{r}\right) \\
\sigma_{1}:\{1, \ldots, p\} \rightarrow\left\{1, \ldots, n_{1}\right\} \backslash \\
\sigma_{2}:\{1, \ldots, p\} \rightarrow\left\{1, \ldots, n_{2}\right\}}} q^{\alpha\left(\sigma_{1}\right)+\beta\left(\sigma_{2}\right)+\operatorname{Cr}(\pi)} \int_{F\left(\sigma_{1}, \sigma_{2}, \pi\right)} f_{1} \otimes f_{2} \otimes \ldots \otimes f_{r} \\
& =\sum_{\substack{\pi \in C_{2}\left(n_{1} \otimes n_{2} \otimes n_{3} \otimes \ldots \otimes n_{r}\right) \\
p_{\pi}=0}} q^{\operatorname{Cr}(\pi)} \int_{\pi} f_{1} \otimes f_{2} \otimes f_{3} \otimes \ldots \otimes f_{r} \\
& +\sum_{p=1}^{\min \left(n_{1}, n_{2}\right)} \sum_{\substack{\pi \in C_{2}\left(\left(n_{1}+n_{2}-2 p\right) \otimes n_{3} \otimes \ldots \otimes n_{r}\right) \\
\sigma_{1}:\{1, \ldots, p\} \rightarrow\left\{1, \ldots, n_{1}\right\} \backslash \\
\sigma_{2}:\{1, \ldots, p\} \rightarrow\left\{1, \ldots, n_{2}\right\}}} q^{\operatorname{Cr}\left(F\left(\sigma_{1}, \sigma_{2}, \pi\right)\right)} \int_{F\left(\sigma_{1}, \sigma_{2}, \pi\right)} f_{1} \otimes f_{2} \otimes \ldots \otimes f_{r} \\
& =\sum_{\substack{\pi \in C_{2}\left(n_{1} \otimes n_{2} \otimes n_{3} \otimes \ldots \otimes n_{r}\right) \\
p_{\pi}=0}} q^{\operatorname{Cr}(\pi)} \int_{\pi} f_{1} \otimes f_{2} \otimes f_{3} \otimes \ldots \otimes f_{r} \\
& +\sum_{p=1}^{\min \left(n_{1}, n_{2}\right)} \sum_{\substack{\pi \in C_{2}\left(n_{1} \otimes n_{2} \otimes n_{3} \otimes \ldots \otimes n_{r}\right) \\
p_{\pi}=p}} q^{\operatorname{Cr}(\pi)} \int_{\pi} f_{1} \otimes f_{2} \otimes f_{3} \otimes \ldots \otimes f_{r} \\
& =\sum_{\pi \in C_{2}\left(n_{1} \otimes n_{2} \otimes n_{3} \otimes \ldots \otimes n_{r}\right)} q^{\mathrm{Cr}(\pi)} \int_{\pi} f_{1} \otimes f_{2} \otimes f_{3} \otimes \ldots \otimes f_{r},
\end{aligned}
$$

which completes the induction procedure.

\section{Fourth moment THEOREM AND APPLiCATIONS}

3.1. Colored pairings. Given $n_{1}, \ldots, n_{R} \geq 1$, we denote by $\operatorname{Col}\left(\left\{n_{1}, \ldots, n_{R}\right\}\right)$ the set of 'colors' in $\left\{n_{1}, \ldots, n_{R}\right\}$, i.e., $\operatorname{Col}\left(\left\{n_{1}, \ldots, n_{R}\right\}\right)=\left\{N_{1}, \ldots, N_{K}\right\}$ where $N_{1}<\ldots<N_{K}$ are such that $\left\{N_{1}, \ldots, N_{k}\right\}=\left\{n_{1}, \ldots, n_{R}\right\}$. We also introduce the set $C_{2}^{\text {col }}\left(n_{i_{1}}, \ldots, n_{i_{r}}\right)$ of pairings in $\mathcal{P}_{2}(\{1, \ldots, r\})$ that respects the coloring of $\left(n_{1}, \ldots, n_{R}\right)$, i.e.,

$$
C_{2}^{c o l}\left(n_{1}, \ldots, n_{R}\right):=\left\{\pi \in \mathcal{P}_{2}(\{1, \ldots, r\}): \text { for all }\{l, m\} \in \pi, n_{l}=n_{m}\right\} .
$$

Given $\pi \in C_{2}^{c o l}\left(n_{1}, \ldots, n_{R}\right)$, we define $\pi_{i}(1 \leq i \leq K)$ as the set of pairs in $\pi$ with the same color $N_{i}$, i.e.,

$$
\pi_{i}:=\left\{\{l, m\} \in \pi: n_{l}=n_{m}=N_{i}\right\} .
$$

Finally, for all subsets $\pi^{\prime}, \pi^{\prime \prime}$ of a given pairing $\pi$, the notation $\operatorname{Cr}\left(\pi^{\prime}, \pi^{\prime \prime}\right)$ will refer to the number of crossings (in $\pi$ ) between pairs of $\pi^{\prime}$ and pairs of $\pi^{\prime \prime}$.

With this notation in hand, we can state the main result of this section.

Theorem 3.1. Fix $q \in[0,1), d \geq 1, n_{1}, \ldots, n_{d} \geq 1$ and consider $d$ sequences $\left\{f_{k}^{1}\right\}_{k \geq 1} \subset$ $L^{2}\left(\mathbb{R}_{+}^{n_{1}}\right), \ldots,\left\{f_{k}^{d}\right\}_{k \geq 1} \subset L^{2}\left(\mathbb{R}_{+}^{n_{d}}\right)$ of symmetric functions. Assume that

$$
\lim _{k \rightarrow \infty}\left\langle f_{k}^{i}, f_{k}^{j}\right\rangle_{q}=c(i, j) \quad \text { for all } i, j \in\{1, \ldots, d\} .
$$


Then, the following four assertions are equivalent as $k \rightarrow \infty$ :

(i) For every $i \in\{1, \ldots, d\}, \varphi_{q}\left(I_{n_{i}}^{X^{(q)}}\left(f_{k}^{i}\right)^{4}\right) \rightarrow\left(2+q^{n_{i}^{2}}\right) c(i, i)^{2}$.

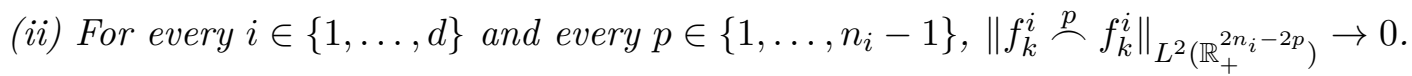

(iii) For every $i \in\{1, \ldots, d\}, I_{n_{i}}^{X^{(q)}}\left(f_{k}^{i}\right) \stackrel{\operatorname{law}}{\rightarrow} \mathcal{G}_{q^{n_{i}^{2}}}(0, c(i, i))$.

(iv) For every $r \geq 1$ and every $i_{1}, \ldots, i_{r} \in\{1, \ldots, d\}$, one has

$$
\varphi_{q}\left(I_{n_{i_{1}}}^{X^{(q)}}\left(f_{k}^{i_{1}}\right) \ldots I_{n_{i_{r}}}^{X^{(q)}}\left(f_{k}^{i_{r}}\right)\right) \rightarrow \sum_{\pi \in C_{2}^{c o l}\left(n_{i_{1}}, \ldots, n_{i_{r}}\right)} \prod_{1 \leq i \leq j \leq K} q^{\operatorname{Cr}\left(\pi_{i}, \pi_{j}\right) N_{i} N_{j}} \prod_{\{l, m\} \in \pi} c\left(i_{l}, i_{m}\right),
$$

where we have set $\left\{N_{1}, \ldots, N_{K}\right\}:=\operatorname{Col}\left(\left\{n_{i_{1}}, \ldots, n_{i_{r}}\right\}\right)$.

Remark that, in the case where the integers $n_{1}, \ldots, n_{d}$ are such that

$$
\operatorname{Card}\left(\operatorname{Col}\left(\left\{n_{1}, \ldots, n_{d}\right\}\right)\right) \geq 2,
$$

it seems difficult to identify the limit in (17) as the distribution of a multidimensional $q^{*}$-Gaussian law for some $q^{*} \in(-1,1)$, due to the (possibly) changing weights $q^{\operatorname{Cr}\left(\pi_{i}, \pi_{j}\right) N_{i}, N_{j}}$.

Before proving Theorem 3.1, let us first explain how it implies Theorem 1.7, Theorem 1.8 and Theorem 1.9.

Proof of Theorem [1.7. Theorem 1.7 is an immediate spin-off of Theorem [3.1] Indeed, take $n_{1}=\ldots=n_{d}=n, f_{k}^{1}=\ldots=f_{k}^{d}=f_{k}$ with $\left\|f_{k}\right\|_{q}^{2} \rightarrow 1$ as $k \rightarrow \infty$. Then $\operatorname{Col}\left(\left\{n_{i_{1}}, \ldots, n_{i_{r}}\right\}\right)=$ $\{n\}, C_{2}^{c o l}\left(n_{i_{1}}, \ldots, n_{i_{r}}\right)=\mathcal{P}_{2}(\{1, \ldots, r\})$, and the limit in (17) becomes $\sum_{\pi \in \mathcal{P}_{2}(\{1, \ldots, r\})} q^{\mathrm{Cr}(\pi) n^{2}}$, which is precisely the $r$ th moment of the $q$-Gaussian law $\mathcal{G}_{q^{n^{2}}}(0,1)$, see indeed (15) and observe that $\mathcal{P}_{2}(\{1, \ldots, r\})=\emptyset$ when $r$ is odd.

Proof of Theorem 1.8. Here again, it is an immediate spin-off of Theorem 3.1, still by considering the case where $n_{1}=\ldots=n_{d}=n$ and $f_{k}^{1}=\ldots=f_{k}^{d}=f_{k}$ with $\left\|f_{k}\right\|_{q}^{2} \rightarrow 1$ as $k \rightarrow \infty$. Indeed, assume that $I_{n}^{X^{(q)}}\left(f_{k}\right)$ converges in law to $\mathcal{G}_{q^{n^{2}}}\left(0, \sigma_{q}^{2}\right)$ for one particular $q \in[0,1]$. Using the implication $(i i i) \Rightarrow(i i)$ in Theorem [3.1, we get that $\left\|f_{k} \stackrel{p}{\sim} f_{k}\right\|_{L^{2}\left(\mathbb{R}_{+}^{2 n-2 p}\right)} \rightarrow 0$ as $k \rightarrow \infty$ for every $p \in\{1, \ldots, n-1\}$. But, since this latter assertion does not depend on $q$, we deduce, by using the converse implication $(i i) \Rightarrow$ (iii) for all the other possible values of $q$, that $I_{n}^{X^{(q)}}\left(f_{k}\right)$ converges in law to $\mathcal{G}_{q^{n^{2}}}\left(0, \sigma_{q}^{2}\right)$ for all $q \in[0,1]$.

Proof of Theorem [1.9. The result for $q=1$ being already known (see [4]), we fix $q \in[0,1$ ) in all the proof. Since our aim is to show a convergence in law, in (17) it is not a loss of generality to replace $G_{l}$ with $I_{1}^{X^{(q)}}\left(e_{l}\right)$, where the sequence $\left\{e_{l}\right\}_{l \in \mathbb{N}} \subset L^{2}\left(\mathbb{R}_{+}\right)$is chosen so that

$$
\int_{0}^{\infty} e_{l}(x) e_{m}(x) d x=\rho(l-m), \quad l, m \in \mathbb{N} .
$$

Indeed, using the fact that the covariance function characterizes the distribution of any centered $q$-Gaussian family, we immediately check that $\left\{G_{l}\right\}_{l \in \mathbb{N}} \stackrel{\text { law }}{=}\left\{I_{1}^{X^{(q)}}\left(e_{l}\right)\right\}_{l \in \mathbb{N}}$. Moreover, such a sequence $\left\{e_{l}\right\}$ is easily shown to exist by considering the linear span $\mathcal{H}$ of $\left\{G_{l}\right\}$. It is indeed a real separable Hilbert space and, consequently, there exists an isometry $\Phi: \mathcal{H} \rightarrow L^{2}\left(\mathbb{R}_{+}\right)$. By setting $e_{l}=\Phi\left(G_{l}\right)$, we get that (18) holds true. 
Now, using (6), we can write

$$
\frac{1}{\sqrt{k}} \sum_{l=0}^{[k t]} H_{n}^{(q)}\left(G_{l}\right) \stackrel{\text { law }}{=} \frac{1}{\sqrt{k}} \sum_{l=0}^{[k t]} H_{n}^{(q)}\left(I_{1}^{X^{(q)}}\left(e_{l}\right)\right)=\frac{1}{\sqrt{k}} \sum_{l=0}^{[k t]} I_{n}^{X^{(q)}}\left(e_{l}^{\otimes n}\right)=I_{n}^{X^{(q)}}\left(\frac{1}{\sqrt{k}} \sum_{l=0}^{[k t]} e_{l}^{\otimes n}\right) .
$$

Fix $d \geq 1$ and $t_{1}, \ldots, t_{d}>0$. For each $i \in\{1, \ldots, d\}$, observe that the kernel

$$
f_{k}\left(t_{i}\right):=\frac{1}{\sqrt{k}} \sum_{l=0}^{\left[k t_{i}\right]} e_{l}^{\otimes n}
$$

is a symmetric function of $L^{2}\left(\mathbb{R}_{+}^{n}\right)$. Moreover, it may be shown (see [10, chapter 7]) that

$$
\lim _{k \rightarrow \infty}\left\langle f_{k}\left(t_{i}\right), f_{k}\left(t_{j}\right)\right\rangle_{q}=\lim _{k \rightarrow \infty} \sum_{\sigma \in \mathfrak{S}_{n}} q^{\operatorname{inv}(\sigma)}\left\langle f_{k}\left(t_{i}\right), f_{k}\left(t_{j}\right)\right\rangle_{L^{2}\left(\mathbb{R}_{+}^{n}\right)}=\sum_{\sigma \in \mathfrak{S}_{n}} q^{\operatorname{inv}(\sigma)} \sum_{l \in \mathbb{Z}} \rho(l)^{n} t_{i} \wedge t_{j}
$$

for all $i, j \in\{1, \ldots, d\}$ (in particular, $\sum_{l \in \mathbb{Z}} \rho(l)^{n} \geq 0$ ) and that

$$
\lim _{k \rightarrow \infty}\left\|f_{k}\left(t_{i}\right) \stackrel{p}{\sim} f_{k}\left(t_{i}\right)\right\|_{L^{2}\left(\mathbb{R}_{+}^{2 n-2 p}\right)}=0
$$

for all $i \in\{1, \ldots, d\}$ and all $p \in\{1, \ldots, n-1\}$. Take $n_{1}=\ldots=n_{d}=n$ in Theorem 3.1 and set $f_{k}^{1}=f_{k}\left(t_{1}\right), \ldots, f_{k}^{d}=f_{k}\left(t_{d}\right)$. Since (ii) holds, we deduce from (17) that, for every $r \geq 1$ and every $s_{1}, \ldots, s_{r} \in\left\{t_{1}, \ldots, t_{d}\right\}$, the quantity

$$
\varphi_{q}\left(I_{n}^{X^{(q)}}\left(f_{k}\left(s_{1}\right)\right) \ldots I_{n}^{X^{(q)}}\left(f_{k}\left(s_{r}\right)\right)\right)=\varphi\left(\frac{1}{\sqrt{k}} \sum_{l=0}^{\left[k s_{1}\right]} H_{n}^{(q)}\left(G_{l}\right) \ldots \frac{1}{\sqrt{k}} \sum_{l=0}^{\left[k s_{r}\right]} H_{n}^{(q)}\left(G_{l}\right)\right)
$$

converges, as $k \rightarrow \infty$, to

$$
\begin{aligned}
& \left(\sum_{\sigma \in \mathfrak{S}_{n}} q^{\operatorname{inv}(\sigma)} \sum_{l \in \mathbb{Z}} \rho(l)^{n}\right)^{r / 2} \sum_{\substack{\pi \in \mathcal{P}_{2}(\{1, \ldots, r\})\\
}} q^{\mathrm{Cr}(\pi) n^{2}} \prod_{\{l, m\} \in \pi} s_{i_{l}} \wedge s_{i_{m}} \\
& =\left(\sum_{\sigma \in \mathfrak{S}_{n}} q^{\operatorname{inv}(\sigma)} \sum_{l \in \mathbb{Z}} \rho(l)^{n}\right)^{r / 2} \varphi_{q_{n}}\left(X^{\left(q_{n}\right)}\left(s_{1}\right) \ldots X^{\left(q_{n}\right)}\left(s_{r}\right)\right),
\end{aligned}
$$

where we set $q_{n}:=q^{n^{2}}$ for simplicity and where $X^{\left(q_{n}\right)}$ is a $q_{n}$-Brownian motion (for the second expression of the limit, see (4) ). This concludes the proof of Theorem 1.9.

We now turn to the proof of Theorem 3.1.

Proof of Theorem 3.1. We shall prove the following sequence of implications: $(i v) \Rightarrow(i i i) \Rightarrow$ $(i) \Rightarrow(i i) \Rightarrow(i v)$.

$(i v) \Rightarrow(i i i) \quad$ Assume $(i v)$, fix $i \in\{1, \ldots, d\}, r \geq 1$ and take $i_{1}=\ldots=i_{r}=i$ in $(i v)$. Let $f^{1}, \ldots, f^{d} \in L^{2}\left(\mathbb{R}_{+}\right)$be such that $\left\langle f^{i}, f^{j}\right\rangle_{L^{2}\left(\mathbb{R}_{+}\right)}=c(i, j)$ for all $i, j \in\{1, \ldots, d\}$. In this case, convergence (17) can be written as

$$
\begin{aligned}
\varphi_{q}\left(I_{n_{i}}^{X^{(q)}}\left(f_{k}^{i}\right)^{r}\right) \rightarrow & \sum_{\substack{\mathcal{P}_{2}(\{1, \ldots, r\})\\
}} q^{\mathrm{Cr}(\pi) n_{i}^{2}} \prod_{\{l, m\} \in \pi}\left\langle f^{i}, f^{i}\right\rangle_{L^{2}\left(\mathbb{R}_{+}\right)} \\
& \sum_{\pi \in \mathcal{P}_{2}(\{1, \ldots, r\})} q^{\mathrm{Cr}(\pi) n_{i}^{2}} \int_{\pi} f^{i} \otimes \ldots \otimes f^{i} \\
& =\varphi_{q_{i}}\left(I_{1}^{X^{\left(q_{i}\right)}}\left(f^{i}\right)^{r}\right) \quad \text { with } q_{i}=q^{n_{i}^{2}},
\end{aligned}
$$


the last equality being an easy consequence of formula (16). Since the convergence in law is equivalent by its very definition to the convergence of all the moments, the implication $(i v) \Rightarrow$ (iii) is shown.

$($ iii $) \Rightarrow(i)$ Obvious (still because convergence in law reduces to convergence of all the moments in our framework).

$(i) \Rightarrow(i i) \quad$ We shall make use of the following proposition.

Proposition 3.2. Fix $q \in(-1,1)$. Then, for every symmetric function $f \in L^{2}\left(\mathbb{R}_{+}^{n}\right)$, we have

$$
\varphi_{q}\left(I_{n}^{X^{(q)}}(f)^{4}\right)=\left(2+q^{n^{2}}\right)\|f\|_{q}^{4}+\sum_{p=1}^{n-1}\left\{\left\|f \stackrel{p}{{ }_{q}} f\right\|_{q}^{2}+\left(\sum_{\sigma \in \mathfrak{S}_{2 n}^{p}} q^{\operatorname{inv}(\sigma)}\right)\|f \stackrel{p}{\underline{f}} f\|_{L^{2}\left(\mathbb{R}_{+}^{2 n-2 p}\right)}^{2}\right\},
$$

where

$$
\mathfrak{S}_{2 n}^{p}=\left\{\sigma \in \mathfrak{S}_{2 n}: \operatorname{Card}(\sigma(\{1, \ldots, n\}) \cap\{1, \ldots, n\})=p\right\} .
$$

Before proving it, let us see how it implies the desired conclusion. Fix $i \in\{1, \ldots, d\}$ and assume that the sequence $\left\{f_{k}^{i}\right\}$ of Theorem 3.1 satisfies $(i)$, that is,

$$
\lim _{k \rightarrow \infty} \varphi_{q}\left(I_{n_{i}}^{X^{(q)}}\left(f_{k}^{i}\right)^{4}\right)=\left(2+q^{n_{i}^{2}}\right) c(i, i)^{2}=\lim _{k \rightarrow \infty}\left(2+q^{n_{i}^{2}}\right)\left\|f_{k}^{i}\right\|_{q}^{2} .
$$

Thanks to (19), we deduce that

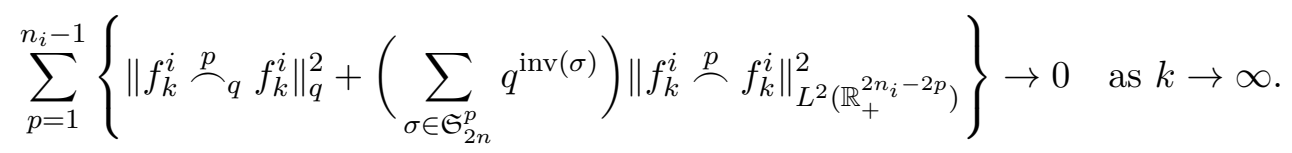

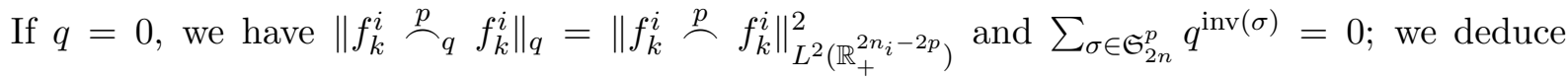
that $\left\|f_{k}^{i} \stackrel{p}{\sim} f_{k}^{i}\right\|_{L^{2}\left(\mathbb{R}_{+}^{2 n_{i}-2 p}\right)}^{2} \rightarrow 0$ for every $p \in\left\{1, \ldots, n_{i}-1\right\}$, which is precisely (ii). If $q>0$, then $\sum_{\sigma \in \mathfrak{S}_{2 n}^{p}} q^{\operatorname{inv}(\sigma)}>0$ and, accordingly, we also get that $\left\|f_{k}^{i} \stackrel{p}{\sim} f_{k}^{i}\right\|_{L^{2}\left(\mathbb{R}_{+}^{2 n_{i}-2 p}\right)}^{2} \rightarrow 0$ for every $p \in\left\{1, \ldots, n_{i}-1\right\}$, that is, $(i i)$ holds true as well.

Proof of Proposition 3.2. By using the product formula (11) together with the isometry (9), we first deduce

$$
\varphi_{q}\left(I_{n}^{X^{(q)}}(f)^{4}\right)=\varphi_{q}\left[\left(\sum_{p=0}^{n} I_{2 n-2 p}\left(f{\stackrel{p}{r_{q}}}_{q} f\right)\right)^{2}\right]=\sum_{p=0}^{n}\left\|f{\stackrel{p}{q_{q}}} f\right\|_{q}^{2} .
$$

Next, observe that $\left(f \stackrel{n}{\stackrel{n}{q}_{q}} f\right)^{2}=\|f\|_{q}^{4}$ and, using (13), write $\|f \otimes f\|_{q}^{2}$ as

$$
\|f \otimes f\|_{q}^{2}=\sum_{\sigma \in \mathfrak{S}_{2 n}} q^{\operatorname{inv}(\sigma)} \int_{P_{2}(\sigma)}(f \otimes f) \otimes(f \otimes f)^{*}=\sum_{p=0}^{n} \sum_{\sigma \in \mathfrak{S}_{2 n}^{p}} q^{\operatorname{inv}(\sigma)} \int_{P_{2}(\sigma)}(f \otimes f) \otimes(f \otimes f)^{*} .
$$

It is readily checked that

$$
\sum_{\sigma \in \mathfrak{S}_{2 n}^{n}} q^{\operatorname{inv}(\sigma)} \int_{P_{2}(\sigma)}(f \otimes f) \otimes(f \otimes f)^{*}=\left(\sum_{\sigma \in \mathfrak{S}_{n}} q^{\operatorname{inv}(\sigma)} \int_{P_{2}(\sigma)} f \otimes f\right)^{2}=\|f\|_{q}^{4},
$$


while

$$
\sum_{\sigma \in \mathfrak{S}_{2 n}^{0}} q^{\operatorname{inv}(\sigma)} \int_{P_{2}(\sigma)}(f \otimes f) \otimes(f \otimes f)^{*}=q^{n^{2}}\left(\sum_{\sigma \in \mathfrak{S}_{n}} q^{\operatorname{inv}(\sigma)} \int_{P_{2}(\sigma)} f \otimes f\right)^{2}=q^{n^{2}}\|f\|_{q}^{4} .
$$

Also, since $f$ is a symmetric function, it is easy to verify that for every $\sigma \in \mathfrak{S}_{2 n}^{p}$ with $p \in$ $\{1, \ldots, n-1\}$, one has

$$
\int_{P_{2}(\sigma)}(f \otimes f) \otimes(f \otimes f)^{*}=\|f \stackrel{p}{\sim} f\|_{L^{2}\left(\mathbb{R}_{+}^{2 n-2 p}\right)}^{2} .
$$

As a result, we deduce that

$$
\|f \otimes f\|_{q}^{2}=\left(1+q^{n^{2}}\right)\|f\|_{q}^{4}+\sum_{p=1}^{n-1}\left(\sum_{\sigma \in \mathfrak{S}_{2 n}^{p}} q^{\operatorname{inv}(\sigma)}\right) \cdot\|f \stackrel{p}{\sim} f\|_{L^{2}\left(\mathbb{R}_{+}^{2 n-2 p}\right)}^{2} .
$$

Remark 3.3. Assume that $n=2$ and let $f \in L^{2}\left(\mathbb{R}_{+}^{2}\right)$ be a symmetric function. It is readily checked that $\left\|f \stackrel{1}{\stackrel{1}{r}_{q}} f\right\|_{q}^{2}=(1+q)^{5}\|f \stackrel{1}{\sim} f\|_{L^{2}\left(\mathbb{R}_{+}^{2}\right)}^{2}$ We deduce from (19) that

$$
\varphi_{q}\left(I_{2}^{X^{(q)}}(f)^{4}\right)=\left(2+q^{4}\right)\|f\|_{q}^{4}+(1+q)^{4}(2 q+1)\|f \stackrel{1}{\sim} f\|_{L^{2}\left(\mathbb{R}_{+}^{2}\right)}^{2} .
$$

In particular, when $q=-1 / 2$ and $f=\sqrt{2} \mathbf{1}_{[0,1]^{2}}$, we have $\varphi_{q}\left(I_{2}^{X^{(q)}}(f)^{2}\right)=\|f\|_{q}^{2}=1$ and $\varphi_{q}\left(I_{2}^{X^{(q)}}(f)^{4}\right)=2+q^{4}$. But $I_{2}^{X^{(q)}}(f)$ is not $\mathcal{G}_{q^{4}}(0,1)$-distributed since, by using the product formula (11) together with the isometry (9) and the fact that $f \stackrel{1}{\stackrel{1}{f}_{q}} f=(1+q)^{2} f \stackrel{1}{\sim} f=$ $\sqrt{2}(1+q)^{2} f$, we have

$$
\begin{aligned}
\varphi_{q}\left(I_{2}^{X^{(q)}}(f)^{3}\right) & =\varphi_{q}\left(I_{2}^{X^{(q)}}(f)\left(I_{4}^{X^{(q)}}(f \otimes f)+I_{2}^{X^{(q)}}(f \stackrel{1}{\sim} q)+\|f\|_{q}^{2}\right)\right) \\
& =\left\langle f, f \stackrel{1}{\sim_{q}} f\right\rangle_{q}=\sqrt{2}(1+q)^{2} \neq 0 .
\end{aligned}
$$

This explicit situation shows that Theorem 3.1 may actually be false when $q$ is negative. However, formula (21) implies the following interesting fact when $n=2$. Let $q \in\left(-1,-\frac{1}{2}\right) \cup\left(-\frac{1}{2}, 1\right]$ and let $\left\{f_{k}\right\}_{k \geq 1}$ be a sequence of symmetric functions in $L^{2}\left(\mathbb{R}_{+}^{2}\right)$ satisfying $\left\|f_{k}\right\|_{q} \rightarrow 1$ as $k \rightarrow \infty$. Then, due to (21) we have equivalence between $(a) \varphi_{q}\left(I_{2}^{X^{(q)}}\left(f_{k}\right)^{4}\right) \rightarrow 2+q^{4}$ and (b) $\left\|f_{k} \stackrel{1}{\sim} f_{k}\right\|_{L^{2}\left(\mathbb{R}_{+}^{2}\right)}^{2} \rightarrow 0$ as $k \rightarrow \infty$. Following the same line of reasoning as in the forthcoming proof of $(i i) \Rightarrow(i v)$, we may deduce that $(a)-(b)$ are also equivalent to $(c) I_{2}^{X^{(q)}}\left(f_{k}\right) \stackrel{\text { law }}{\rightarrow} \mathcal{G}_{q^{4}}(0,1)$. That is, when $n=2$, Theorem 3.1 happens to hold true for all $q \in\left(-1,-\frac{1}{2}\right) \cup\left(-\frac{1}{2}, 1\right]$.

$(i i) \Rightarrow(i v) \quad$ Assume $(i i)$ and let us show (iv). Our starting point is the formula (16), which yields

$$
\varphi_{q}\left(I_{n_{i_{1}}}^{X^{(q)}}\left(f_{k}^{i_{1}}\right) \ldots I_{n_{i_{r}}}^{X^{(q)}}\left(f_{k}^{i_{r}}\right)\right)=\sum_{\pi \in C_{2}\left(n_{i_{1}} \otimes \ldots \otimes n_{i_{r}}\right)} q^{\operatorname{Cr}(\pi)} \int_{\pi} f_{k}^{i_{1}} \otimes \ldots \otimes f_{k}^{i_{r}} .
$$

Let us introduce the subset $C_{2}^{0}\left(n_{i_{1}} \otimes \ldots \otimes n_{i_{r}}\right)$ of pairings $\pi \in C_{2}\left(n_{i_{1}} \otimes \ldots \otimes n_{i_{r}}\right)$ that meet the following two conditions:

(A): If two blocks of $n_{i_{1}} \otimes \ldots \otimes n_{i_{r}}$ are linked, then they necessarily have the same cardinality. (B): Any two blocks of $n_{i_{1}} \otimes \ldots \otimes n_{i_{r}}$ having the same number $n$ of elements are necessarily linked by 0 or $n$ pair(s). 
Let $\pi \in C_{2}\left(n_{i_{1}} \otimes \ldots \otimes n_{i_{r}}\right) \backslash C_{2}^{0}\left(n_{i_{1}} \otimes \ldots \otimes n_{i_{r}}\right)$ and assume that $\pi$ does not satisfy (A), i.e., that there exists two blocks (say the $l$ th and the $m$ th blocks) with different cardinalities $\left(n_{i_{l}}<n_{i_{m}}\right)$ that are connected in $\pi$ by $p$ pairs, for some $p \in\left\{1, \ldots, n_{i_{l}}\right\}$. Then there exists $\pi^{\prime} \in C_{2}\left(\left(n_{i_{l}}+n_{i_{m}}-2 p\right) \otimes n_{i_{1}} \otimes \ldots \otimes \widehat{n_{i_{l}}} \otimes \ldots \otimes \widehat{n_{i_{m}}} \otimes \ldots \otimes n_{i_{r}}\right)$ such that

$$
\int_{\pi} f_{k}^{i_{1}} \otimes \ldots \otimes f_{k}^{i_{r}}=\int_{\pi^{\prime}}\left(f_{k}^{i_{l}} \stackrel{p}{ } f_{k}^{i_{m}}\right) \otimes f_{k}^{i_{1}} \otimes \ldots \otimes \widehat{f_{k}^{i_{l}}} \otimes \ldots \otimes \widehat{f_{k}^{i_{m}}} \otimes \ldots \otimes f^{i_{r}},
$$

where the notation ${ }^{\wedge}$ means that this particular object is removed. By using Cauchy-Schwarz, we get

$$
\left|\int_{\pi} f_{k}^{i_{1}} \otimes \ldots \otimes f_{k}^{i_{r}}\right| \leq\left\|f_{k}^{i_{l}} \stackrel{p}{\stackrel{i^{\prime}}{i_{m}}} f_{k}^{i_{m}\left(\mathbb{R}_{+}^{n_{i_{l}}+n_{i_{m}}-2 p}\right)} \prod_{s \in\{1, \ldots, r\} \backslash\{l, m\}}\right\| f_{k}^{i_{s}} \|_{L^{2}\left(\mathbb{R}_{+}^{n_{i_{s}}}\right)} .
$$

Now, some elementary computations yield

$$
\begin{aligned}
\left\|f_{k}^{i_{l}} \stackrel{p}{\sim} f_{k}^{i_{m}}\right\|_{L^{2}\left(\mathbb{R}_{+}^{n_{i_{l}}+n_{i_{m}}-2 p}\right)}^{2} & =\left\langle f_{k}^{i_{l}} \stackrel{n_{i_{l}}-p}{\complement} f_{k}^{i_{l}}, f_{k}^{i_{m}} \stackrel{n_{i_{m}}-p}{\sim} f_{k}^{i_{m}}\right\rangle_{L^{2}\left(\mathbb{R}_{+}^{2 p}\right)} \\
& \leq\left\|f_{k}^{i_{l}} \stackrel{n_{i_{l}}-p}{\frown} f_{k}^{i_{l}}\right\|_{L^{2}\left(\mathbb{R}_{+}^{2 p}\right)}\left\|f_{k}^{i_{m}} \stackrel{n_{i_{m}}-p}{\sim} f_{k}^{i_{m}}\right\|_{L^{2}\left(\mathbb{R}_{+}^{2 p}\right)} .
\end{aligned}
$$

Since $n_{i_{m}}-p \in\left\{1, \ldots, n_{i_{m}}-1\right\}$, we know that $\left\|f_{k}^{i_{m}} \stackrel{n_{i_{m}}-p}{\sim} f_{k}^{i_{m}}\right\|_{L^{2}\left(\mathbb{R}_{+}^{2 p}\right)} \rightarrow 0$ as $k \rightarrow \infty$, and accordingly $\int_{\pi} f_{k}^{i_{1}} \otimes \ldots \otimes f_{k}^{i_{r}} \rightarrow 0$.

On the other hand, let $\pi \in C_{2}\left(n_{i_{1}} \otimes \ldots \otimes n_{i_{r}}\right) \backslash C_{2}^{0}\left(n_{i_{1}} \otimes \ldots \otimes n_{i_{r}}\right)$ be an element not satisfying (B), i.e., such that there exists two blocks (say the $l$ th and the $m$ th blocks) with the same cardinality $n$ that are connected in $\pi$ by $p$ pairs, for some $p \in\{1, \ldots, n-1\}$. Using similar arguments as above, we get

$$
\left|\int_{\pi} f_{k}^{i_{1}} \otimes \ldots \otimes f_{k}^{i_{r}}\right|^{2} \leq\left\|f_{k}^{i_{l}} \stackrel{n-p}{\leftrightharpoons} f_{k}^{i_{l}}\right\|_{L^{2}\left(\mathbb{R}_{+}^{2 p}\right)}\left\|f_{k}^{i_{m}} \stackrel{n-p}{\leftrightharpoons} f_{k}^{i_{m}}\right\|_{L^{2}\left(\mathbb{R}_{+}^{2 p}\right)} \prod_{s \in\{1, \ldots, r\} \backslash\{l, m\}}\left\|f_{k}^{i_{s}}\right\|_{L^{2}\left(\mathbb{R}_{+}^{n_{i_{s}}}\right)}^{2},
$$

and, since $n-p \in\{1, \ldots, n-1\}$, we can here again conclude that $\int_{\pi} f_{k}^{i_{1}} \otimes \ldots \otimes f_{k}^{i_{2 r}} \rightarrow 0$.

Going back to (22), we deduce that

$$
\varphi_{q}\left(I_{n_{i_{1}}}^{X^{(q)}}\left(f_{k}^{i_{1}}\right) \ldots I_{n_{i_{r}}}^{X^{(q)}}\left(f_{k}^{i_{r}}\right)\right)-\sum_{\pi \in C_{2}^{0}\left(n_{i_{1}} \otimes \ldots \otimes n_{i_{r}}\right)} q^{\operatorname{Cr}(\pi)} \int_{\pi} f_{k}^{i_{1}} \otimes \ldots \otimes f_{k}^{i_{r}} \rightarrow 0
$$

as $k \rightarrow \infty$. Now, with every $\pi \in C_{2}^{0}\left(n_{i_{1}} \otimes \ldots \otimes n_{i_{r}}\right)$, we associate a pairing $\widetilde{\pi} \in \mathcal{P}_{2}(\{1, \ldots, r\})$ according to the basic following procedure: two points $l<m \in\{1, \ldots, r\}$ are linked in $\tilde{\pi}$ if the $l$ th and $m$ th blocks of $n_{i_{1}} \otimes \ldots \otimes n_{i_{r}}$ are linked in $\pi$ (see Figure 2). Owing to the two conditions (A) and (B), it is clear that $\widetilde{\pi}$ defines an element of $C_{2}^{c o l}\left(n_{i_{1}}, \ldots, n_{i_{r}}\right)$. Then, divide $\tilde{\pi}$ into the disjoint subsets $\tilde{\pi}_{1}, \ldots, \tilde{\pi}_{K}$, where $\tilde{\pi}_{t}$ stands for the set of pairs having the same color $N_{t}$. In the sequel, we will use the following explicit notation

$$
\tilde{\pi}_{t}=\left\{\left\{l_{s}^{(t)}, m_{s}^{(t)}\right\}, l_{s}^{(t)}<m_{s}^{(t)}, 1 \leq s \leq R_{t}\right\} \quad, \quad t \in\{1, \ldots, K\} .
$$

Conversely, it is clear that any pairing $\pi \in C_{2}^{0}\left(n_{i_{1}} \otimes \ldots \otimes n_{i_{r}}\right)$ can be reconstructed from the two following ingredients:

- its "projection" $\widetilde{\pi}$,

- the description of the links in $\pi$ coded by a single pair in $\widetilde{\pi}$. This description can be made clear through a set of permutations $\sigma_{s}^{(t)} \in \mathfrak{S}_{N_{t}}$ associated with each pair $\left(l_{s}^{(t)}, m_{s}^{(t)}\right)$ of $\widetilde{\pi}$, according 
to the following principle: in $\pi$, the links between the $l_{s}^{(t)}$ th and $m_{s}^{(t)}$ th blocks are given (when considering these blocks isolated from the others) by the set

$$
\left\{\left(N_{t}+1-i, N_{t}+\sigma_{s}^{(t)}(i)\right), 1 \leq i \leq N_{t}\right\} .
$$

With this identification in mind, our computation of $\operatorname{Cr}(\pi)$ for $\pi \in C_{2}^{0}\left(n_{i_{1}} \otimes \ldots \otimes n_{i_{r}}\right)$ relies on the two following observations:

- a crossing in $\widetilde{\pi}$ between a pair in $\widetilde{\pi}_{i}$ and a pair in $\widetilde{\pi}_{j}$ gives birth to $N_{i} N_{j} \operatorname{crossings~in} \pi$.

- the number of crossings in $\pi$ between the pairs that connect the $l_{s}^{(t)}$ th and $m_{s}^{(t)}$ th blocks is given by $\operatorname{inv}\left(\sigma_{s}^{(t)}\right)$.

From the above considerations we deduce the following formula: for every fixed $\pi \in C_{2}^{0}\left(n_{i_{1}} \otimes\right.$ $\left.\cdots \otimes n_{i_{r}}\right)$, one has

$$
q^{\mathrm{Cr}(\pi)} \int_{\pi} f_{k}^{i_{1}} \otimes \ldots \otimes f_{k}^{i_{r}}=\prod_{1 \leq i \leq j \leq K} q^{\mathrm{Cr}\left(\widetilde{\pi}_{i}, \tilde{\pi}_{j}\right) N_{i} N_{j}} \prod_{1 \leq t \leq K} \prod_{1 \leq s \leq R_{t}} q^{\mathrm{inv}\left(\sigma_{s}^{(t)}\right)} \int_{P_{2}\left(\sigma_{s}^{(t)}\right)} f_{k}^{i_{l}^{l_{s}^{(t)}}} \otimes f_{k}^{i_{s}^{(t)}} .
$$

Therefore,

$$
\begin{aligned}
& \sum_{\pi \in C_{2}^{0}\left(n_{i_{1}} \otimes \ldots \otimes n_{i_{r}}\right)} q^{\mathrm{Cr}(\pi)} \int_{\pi} f_{k}^{i_{1}} \otimes \ldots \otimes f_{k}^{i_{r}} \\
& \quad=\sum_{\tilde{\pi} \in C_{2}^{\text {col }}\left(n_{i_{1}}, \ldots, n_{i_{r}}\right)} \prod_{1 \leq i \leq j \leq K} q^{\mathrm{Cr}\left(\tilde{\pi}_{i}, \tilde{\pi}_{j}\right) N_{i} N_{j}} \prod_{1 \leq t \leq K} \prod_{\{l, m\} \in \tilde{\pi}_{t}} \sum_{\sigma \in \mathfrak{S}_{N_{t}}} q^{\operatorname{inv}(\sigma)} \int_{P_{2}(\sigma)} f_{k}^{i_{l}} \otimes f_{k}^{i_{m}} .
\end{aligned}
$$

At this point, let us recall that

$$
\sum_{\sigma \in \mathfrak{S}_{N_{t}}} q^{\operatorname{inv}(\sigma)} \int_{P_{2}(\sigma)} f_{k}^{i_{l}} \otimes f_{k}^{i_{m}}=\left\langle f_{k}^{i_{l}}, f_{k}^{i_{m}}\right\rangle_{q} \rightarrow c\left(i_{l}, i_{m}\right)
$$

as $k \rightarrow \infty$, so that

$\sum_{\pi \in C_{2}^{0}\left(n_{i_{1}} \otimes \ldots \otimes n_{i_{r}}\right)} q^{\mathrm{Cr}(\pi)} \int_{\pi} f_{k}^{i_{1}} \otimes \ldots \otimes f_{k}^{i_{r}} \rightarrow \sum_{\pi \in C_{2}^{c o l}\left(\left(n_{i_{1}}, \ldots, n_{i_{r}}\right)\right)} \prod_{1 \leq i \leq j \leq K} q^{\operatorname{Cr}\left(\pi^{i}, \pi^{j}\right) N_{i} N_{j}} \prod_{\{l, m\} \in \pi} c\left(i_{l}, i_{m}\right)$

as $k \rightarrow \infty$ which, thanks to (23), entails (17). The proof of Theorem 3.1 is complete.

\section{REFERENCES}

[1] P. Biane and R. Speicher (1998). Stochastic calculus with respect to free Brownian motion and analysis on Wigner space. Probab. Theory Rel. Fields 112, 373-409.

[2] M. Bożejko and R. Speicher (1991). An example of a generalized Brownian motion. Comm. Math. Phys. 137, 519-531.

[3] M. Bożejko and R. Speicher (1996). Interpolations between bosonic and fermionic relations given by generalized Brownian motions. Math. Z. 222, 135-160.

[4] P. Breuer and P. Major (1983). Central limit theorems for non-linear functionals of Gaussian fields. J. Mult. Anal. 13, 425-441.

[5] C. Donati-Martin (2003). Stochastic integration with respect to $q$-Brownian motion. Probab. Theory Rel. Fields 125, 77-95.

[6] U. Frisch and R. Bourret (1970). Parastochastics. J. Math. Phys. 11, 364-390.

[7] O. W. Greenberg (1991). Particles with small violations of Fermi or Bose statistics. Phys. Rev. D 43, 4111-4120. 

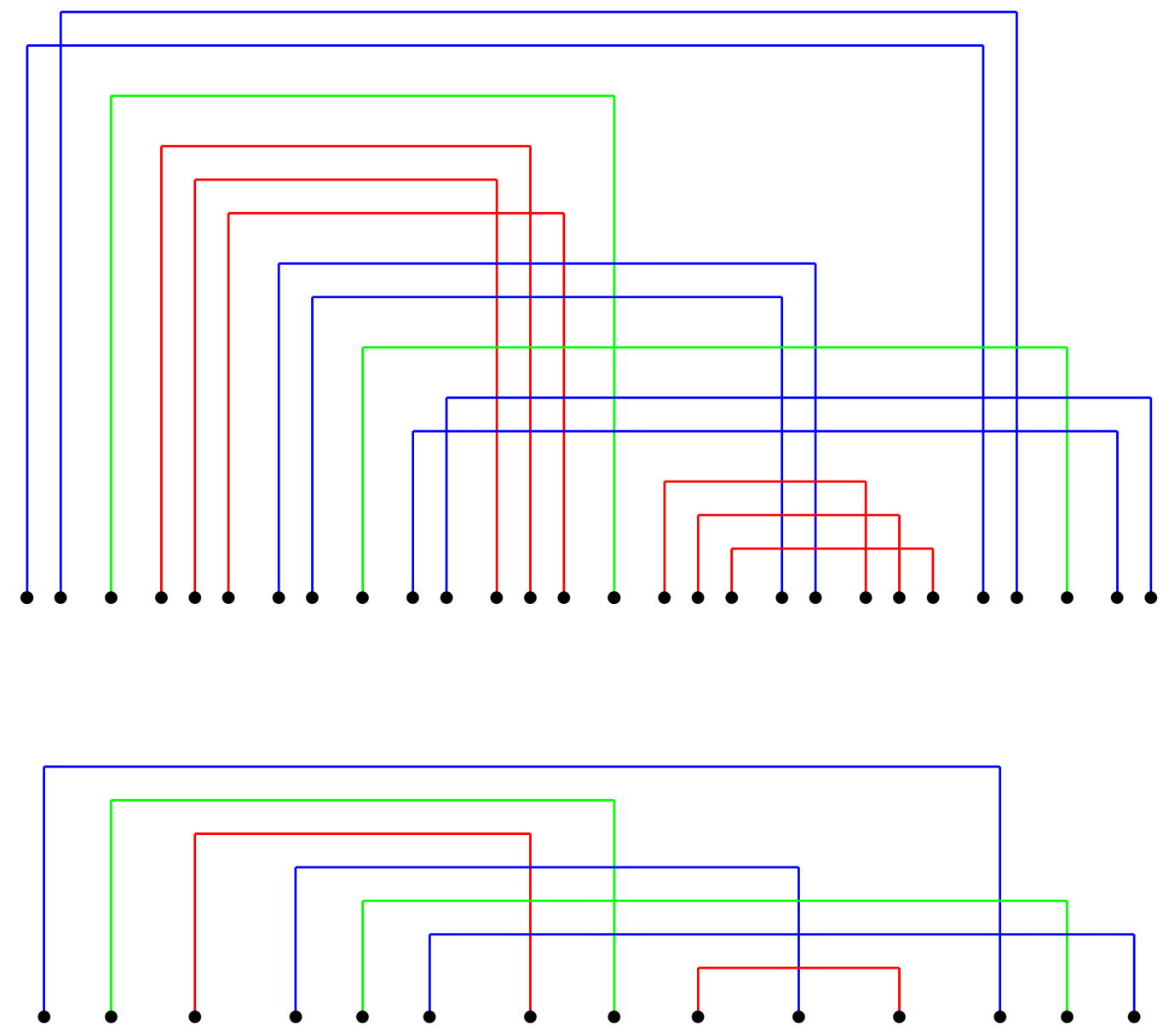

Figure 2. A pairing $\pi$ in $C_{2}^{0}(2 \otimes 1 \otimes 3 \otimes 2 \otimes 1 \otimes 2 \otimes 3 \otimes 1 \otimes 3 \otimes 2 \otimes$ $3 \otimes 2 \otimes 1 \otimes 2$ ) (above) projected in $\tilde{\pi}$ (below). Here, $\tilde{\pi}_{1}=\{(2,8),(5,13)\}$, $\tilde{\pi}_{2}=\{(1,12),(4,10),(6,14)\}, \tilde{\pi}_{3}=\{(3,7),(9,11)\}$.

[8] T. Kemp, I. Nourdin, G. Peccati and R. Speicher (2011). Wigner chaos and the fourth moment. Ann. Probab., in press.

[9] A. Nica and R. Speicher (2006). Lectures on the Combinatorics of Free Probability. Cambridge University Press.

[10] I. Nourdin and G. Peccati (2012). Normal Approximations Using Malliavin Calculus: from Stein's Method to Universality. Cambridge Tracts in Mathematics. Cambridge University Press.

[11] D. Nualart (2006). The Malliavin calculus and related topics. Springer Verlag, Berlin, Second edition.

[12] D. Nualart and G. Peccati (2005). Central limit theorems for sequences of multiple stochastic integrals. Ann. Probab. 33 (1), 177-193.

[13] P. Sniady (2001). Gaussian random matrix models for $q$-deformed Gaussian variables. Comm. Math. Phys. 216, 515-537.

[14] D.V. Voiculescu (1991). Limit laws for random matrices and free product. Invent. Math. 104, 201220. 